\title{
A novel biclustering approach with iterative optimization to analyze gene expression data
}

This article was published in the following Dove Press journal:

Advances and Applications in Bioinformatics and Chemistry

6 September 2012

Number of times this article has been viewed

\section{Sawannee Sutheeworapong ${ }^{1,2}$ \\ Motonori Ota ${ }^{4}$ \\ Hiroyuki Ohta' \\ Kengo Kinoshita ${ }^{2,3}$ \\ 'Department of Biological Sciences, Graduate School of Biosciences and Biotechnology, Tokyo Institute of Technology, Tokyo, Japan; ${ }^{2}$ Graduate School of Information Sciences, ${ }^{3}$ Institute of Development, Aging and Cancer, Tohoku University, Miyagi, Japan; ${ }^{4}$ Graduate School of Information Sciences, Nagoya University, Nagoya, Japan}

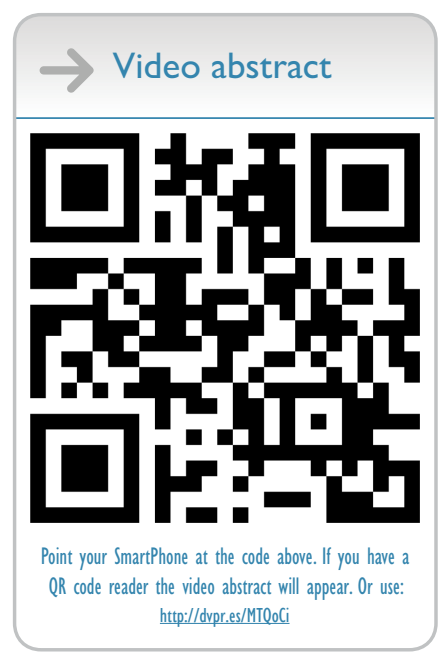

Correspondence: Kengo Kinoshita Laboratory of Systems Bioinformatics, Graduate School of Information Science, Tohoku University, Aoba-ku, Sendai, 980-8579, Japan

Email kengo@ecei.tohoku.ac.jp
Objective: With the dramatic increase in microarray data, biclustering has become a promising tool for gene expression analysis. Biclustering has been proven to be superior over clustering in identifying multifunctional genes and searching for co-expressed genes under a few specific conditions; that is, a subgroup of all conditions. Biclustering based on a genetic algorithm (GA) has shown better performance than greedy algorithms, but the overlap state for biclusters must be treated more systematically.

Results: We developed a new biclustering algorithm (binary-iterative genetic algorithm [BIGA]), based on an iterative GA, by introducing a novel, ternary-digit chromosome encoding function. BIGA searches for a set of biclusters by iterative binary divisions that allow the overlap state to be explicitly considered. In addition, the average of the Pearson's correlation coefficient was employed to measure the relationship of genes within a bicluster, instead of the mean square residual, the popular classical index. As compared to the six existing algorithms, BIGA found highly correlated biclusters, with large gene coverage and reasonable gene overlap. The gene ontology (GO) enrichment showed that most of the biclusters are significant, with at least one GO term over represented.

Conclusion: BIGA is a powerful tool to analyze large amounts of gene expression data, and will facilitate the elucidation of the underlying functional mechanisms in living organisms.

Keywords: biclustering, microarray data, genetic algorithm, Pearson's correlation coefficient

\section{Background}

The complete sequencing of the genomes of many organisms has led to the launch of various omics studies. In one study, the advent of deoxyribonucleic acid (DNA) microarray technology has enabled the monitoring of the expression levels of numerous genes at a time, under many different growth conditions. This technique is now widely used in diverse types of biological research, such as identifying disease markers, reconstructing cellular signaling pathways, and inferring gene regulatory networks. DNA microarray technology has also provided numerous biological insights. ${ }^{1-3}$ Data generated from even a few array measurements are quite complex, and the amounts of microarray data available in public databases are dramatically increasing, due to the efficiency and rapid improvement of DNA microarray technologies. As a result, the interpretation of DNA microarray data obtained under a large number of conditions has become a challenging problem.

In the analyses of a large dataset, as the first step, researchers usually search for similar patterns appearing within the data. In the case of DNA microarray data, similar patterns of gene expression data are often investigated by using cluster analyses, such as K-means 
clustering ${ }^{4}$ and hierarchical clustering. ${ }^{5}$ Although clustering can provide considerable biological information, conventional clustering algorithms may not be suitable for some analyses of microarray data for the following two reasons. Firstly, there are many genes that encode proteins involved in several functional activities at a time, but the conventional clustering methods cannot identify these genes, because they only allow a gene to belong to one cluster at a time, instead of multiple clusters. Secondly, it is difficult to find the genes that are co-expressed under a few specific conditions but are differently expressed under other conditions because the similarity of the genes in conventional clustering is determined by the entire expression data. ${ }^{6,7}$

In terms of the above shortcomings, biclustering is more effective than conventional clustering, since it can cluster both genes and conditions simultaneously, and a gene (or a condition) can be involved in multiple clusters at a time. ${ }^{7}$ The concept of biclustering was first proposed by Hartigan, ${ }^{8}$ and Cheng and Church $^{9}$ applied it to search for the most homogeneously expressed genes over certain sets of conditions by using greedy search algorithms. ${ }^{9}$ Most biclustering algorithms have been implemented with greedy search algorithms, ${ }^{1,10,11}$ to reduce the calculation costs. One such bicluster, a maximum bicluster, is known as a nondeterministic polynomial time (NP)-complete problem that can possibly be solved in polynomial time using a nondeterministic Turing machine, ${ }^{12}$ and a greedy search algorithm is required for actual applications to provide efficient approximations. Usually, one greedy search results in one bicluster, and the greedy search approach is repeatedly applied to the data, while preventing the reproduction of similar biclusters. The greedy search then tries to obtain a set of various biclusters as the final output.

Biclustering has also been implemented by using a genetic algorithm (GA) to find a practical solution to balance bicluster quality and calculation cost. A GA emulates an evolutionary processes to obtain nearly optimal solutions. ${ }^{13}$ Initially, a set of candidate solutions is prepared; each solution being called a chromosome. The chromosomes evolve by exchanging their parts and changing some elements into a different state, and elite chromosomes are selected to survive as the parents of the next generation. This evolution and selection process is repeated over a number of generations to yield an optimal solution. ${ }^{13}$ Bleuler et $a{ }^{14}$ first applied GA to biclustering, whereby a binary string (representing a gene or a condition belonging to a bicluster, or not) was employed as a representation of chromosomes. To avoid any redundancy of the resulting biclusters, Bleuler et al introduced a special selection operator called environment selection. Chakraborty and Maka ${ }^{15}$ have generated a similar GA-based biclustering, but different in terms of chromosome initialization. Initial chromosomes are prepared by K-means clustering. These methods find an optimum set of biclusters from one GA search. For such methods, it would be difficult to obtain a set of various, nonredundant biclusters, because only better chromosomes can survive by the selection process of GA, and thus the resulting biclusters tend to converge into similar results in the later generations. ${ }^{14,15}$ Another type of GA-based biclustering, Sequential Evolutionary Biclustering (SEBI), has a distinct strategy. SEBI initially applies GA to select the optimal bicluster, and then this process is repeated so that the genes and the conditions in the biclusters already selected are less likely to be selected again. In other words, although SEBI would generate a set of diverse biclusters, it de-empathizes the overlap of biclusters, a significant feature of biclustering. ${ }^{16}$

In the present study, we propose BIGA as the basis of a novel biclustering approach. In BIGA, an attempt is made to progressively divide the large amounts of input data into small datasets, by iteratively using GA, such as SEBI. Instead of evaluating a set of biclusters, GA is applied to each division process. Therefore, the resulting biclusters are substantially diverse. In addition, BIGA introduces the overlap state explicitly defined in the ternary digit (or trit) encoding chromosome. In this study, the algorithm is described, the performance of BIGA is compared with those of six existing biclustering algorithms, and the biological relevance of BIGA is evaluated by using gene ontology (GO) enrichment analyses. Finally, we conclude that BIGA is a powerful and practical solution for biclustering with highdimensional data.

\section{Material and methods Definition of biclusters}

BIGA accepts a set of gene expression data with the matrix form $D=(G, C)$, including $N$ rows of genes $G=\left\{g_{1}, g_{2}, \ldots, g_{N}\right\}$ and $M$ columns of conditions or samples $C=\left\{c_{1}, c_{2}, \ldots, c_{M}\right\}$, where $N$ and $M$ are the total numbers of genes and conditions, respectively. All genes will be clustered into $K$ overlapping biclusters $B=\left\{B_{1}, B_{2}, \ldots, B_{K}\right\}$, and each bicluster $\left(B_{i}\right)$ corresponds to a submatrix $B_{i}=(X, Y)$ of $D$, where $X \subseteq G$ and $Y \subseteq C$. The sizes of $X$ and $Y$, ie, the numbers of genes and the conditions of a bicluster, are denoted by $n$ and $m$, in which $n \leq N$ and $m \leq M$, respectively.

\section{Binary-iterative genetic algorithm}

In order to decompose $D$ into $B$ systematically, a binary tree was introduced. Generally, a binary tree comprises nodes 
and directed edges, in which each node can be extended to at most two child nodes. ${ }^{17}$ In this work, we regarded each bicluster and each edge as a node and a parent-child relationship between a bicluster pair, respectively. We designated the method as BIGA.

BIGA consists of the following three steps. A schematic diagram of BIGA is shown in (Figure 1).

Step 1: A division of microarray data is represented by a string, a sequence of trit $(0,1,2)$ with the length of $n$ (number of genes in the parent bicluster) $+m$ (number of conditions in the parent bicluster). The trit 0,1 , and 2 means that an associated gene or condition is contained in either of two biclusters, $b_{\text {left }}$ or $b_{\text {right }}$, or both, respectively. This means that one string can encode the division of one bicluster into two biclusters, while allowing overlap. An example of this encoding is shown in (Figure 1A). The "|" symbol serves as a spacer of the genes and conditions for clarity. The string is equivalent to the division illustrated by the matrix (microarray data, or a bicluster) in the middle of (Figure 1A). In the matrix, the rows and the columns correspond to the genes and the conditions, respectively. The cell of the matrix belongs to either $b_{\text {left }}$ (blue cell), $b_{\text {right }}$ (red), or both (violet), under the decoding rule shown in (Figure 1B). The white cells are ignored because they are not coexpressed with color cells. Consequently, the bicluster shown in the middle of (Figure 1A) represents the division into two biclusters on the right of (Figure 1A).

Step 2: To search for the best chromosome (the best trit string) representing the optimal division of a bicluster, GA is performed (rectangles in Figure 1C). In the GA procedure, a mutation and a crossover are introduced into each chromosome. Each number on a chromosome is altered to 0,1 , or 2 , for the mutation; whereas two chromosomes exchange corresponding parts with each other in the crossover. Chromosomes with higher fitness scores (described in the following section) survive in the next generation, and all other chromosomes are discarded. GA was implemented via Java Genetic Algorithm Product, ${ }^{18}$ with a mutation rate of 0.01 and a crossover rate of 0.5 . Finally, the best chromosome after 100 generations of GA (the underlined string in the rectangle) is selected, based on the fitness score (see the next section). The best chromosome is then decoded into two biclusters $\left(b_{\text {left }}\right.$ and $\left.b_{\text {right }}\right)$. We decide whether to continue with further decompositions after the evaluation of the biclusters, as follows.

Step 3: Evaluation of biclusters. For each child bicluster, the numbers of genes and conditions, the average Pearson's correlation coefficient (PCC), and the parent-child
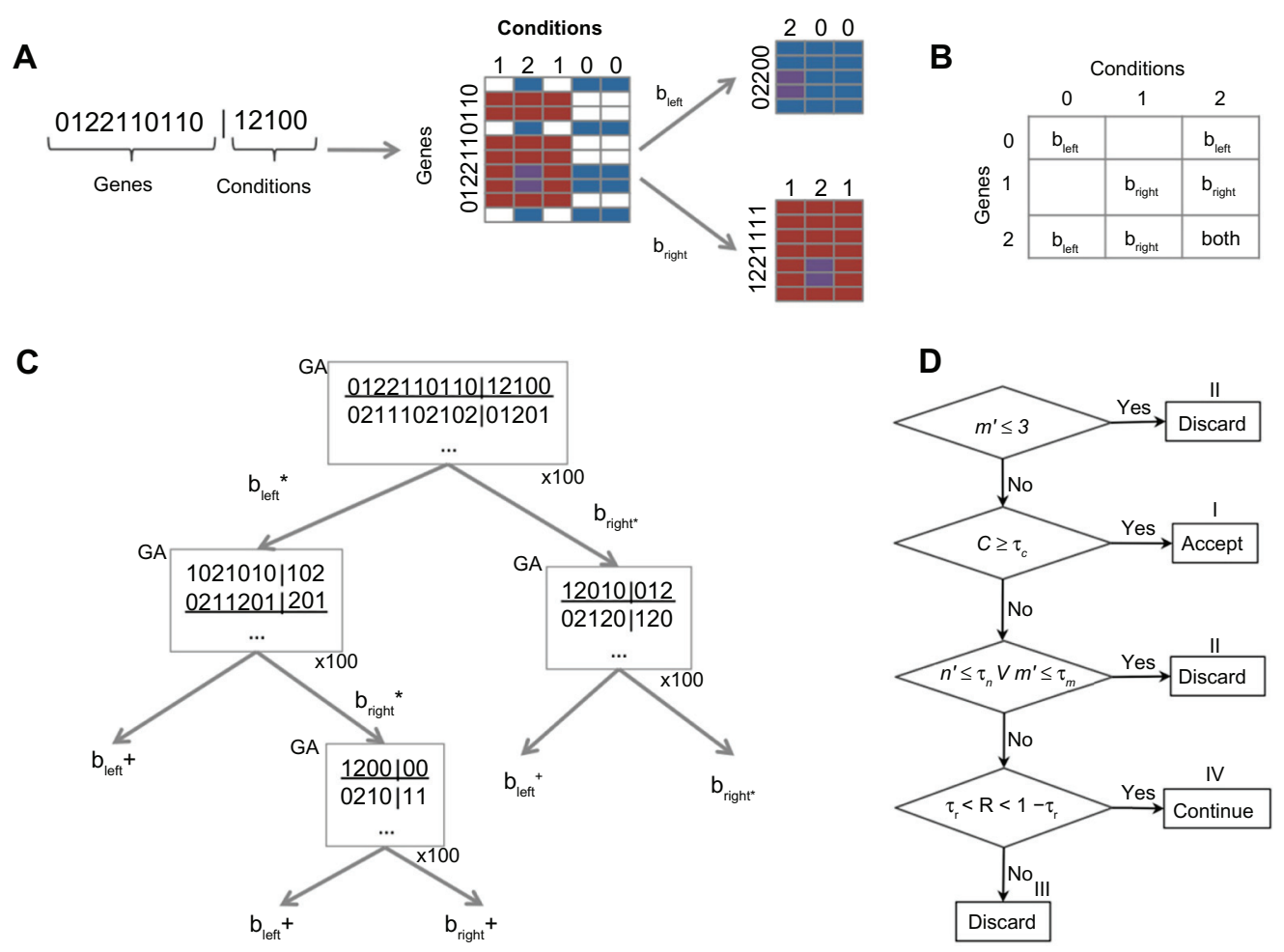

Figure I Schematic diagram of binary-iterative genetic algorithm. (A) Decomposition of a parent bicluster into two child biclusters encoded in a string (left panel). The string indicates that a parent bicluster (middle panel) is divided into two child biclusters (right panel). The red, blue, and violet cells in the biclusters belong to $b_{\text {left }} \mathrm{b}_{\text {right }}$, and both, respectively. (B) Decoding rule of a string. (C) Binary division performed by genetic algorithm (GA). The best string is underlined in the rectangle. For each GA, the generated biclusters $\left(b_{\text {left }}\right.$ and $\left.b_{\text {right }}\right)$ are evaluated to determine their states: continue the decomposition $(*)$, quit the decomposition and accept $(+)$, or quit the decomposition and discard (-). (D) Flow diagram of the bicluster evaluation. 
redundancy are examined to decide whether we should quit or continue the decomposition. Subsequently, the bicluster is either accepted as an element of the final biclusters, $B$, or discarded. We calculate the PCC of every gene pair in a bicluster, and average them (the average PCC). The parent-child redundancy is defined as the ratio of the number of genes of the child bicluster $\left(n^{\prime}\right)$ to that of the parent bicluster $(n)$. Therefore, a small parent-child redundancy indicates that the child bicluster contains a smaller number of genes than the parent, and a large parent-child redundancy means that the number of genes in the child bicluster is almost the same as that of the parent. The average PCC and the parent-child redundancy are abbreviated as $C$ and $R$, respectively. The decision process is illustrated in (Figure 1D). Briefly, the process employs four rules: (I) we quit the decomposition and accept the bicluster if $C$ is higher than the threshold $\tau_{c}$. (II) we quit the decomposition and discard the bicluster if the bicluster is "small," which is judged by the thresholds $\tau_{n}$ and $\tau_{m}$ for $n^{\prime}$ and $m^{\prime}$, respectively. (III) we also quit the decomposition and discard the bicluster if the redundancy, $R$, is small $\left(R<\tau_{r}\right)$ or large $\left(\mathrm{R}>1-\tau_{r}\right)$. The latter rule was employed to reduce the calculation cost, because a child bicluster that is similar to its parent bicluster and has a low $C$ is not considered to produce promising results. Using the forth rule: (IV) we continue the decomposition. Four thresholds, $\tau_{n}, \tau_{m}, \tau_{c}$, and $\tau_{r}$, were empirically determined as $30,10,0.65$, and 0.15 , respectively (see Table S1). The Greek symbols in (Figure 1D) indicate the rule applied in each decision. In (Figure 1C), the accepted and discarded biclusters are marked by + and - symbols. The bicluster to be decomposed is marked by a * symbol. Figure $1 \mathrm{C}$ indicates that four biclusters are accepted.

\section{Fitness function}

In general, large biclusters including co-expressed genes across many specific conditions are preferable. The average PCC of a bicluster was employed to evaluate the gene coexpression. Furthermore, the relative area $A$ of the bicluster, defined by $\left(n^{\prime} / n\right)^{\alpha}\left(m^{\prime} / m\right)^{\beta}$, using the gene and condition numbers of the parent and child biclusters was used to evaluate the size of a bicluster. Two parameters were introduced for gene-weight $(\alpha)$ and condition-weight $(\beta)$, to control the balance between the number of genes and that of the conditions $(0<\alpha, \beta<1)$ in a relative area, $A$. The fitness function of a chromosome was defined as follows (Equation 1):

$$
f(c)=A\left(b_{\text {left }}\right) C\left(b_{\text {left }}\right)+A\left(b_{\text {right }}\right) C\left(b_{\text {right }}\right),
$$

where $c, b_{i}(i=$ left or right), $A(b)$, and $C(b)$ denote a chromosome, one of the child biclusters, the relative area of child bicluster $b$, and the average PCC of child bicluster $b$, respectively.

The balance between $\alpha$ and $\beta$ was important in order to select biologically meaningful biclusters when using $f(c)$. Since a high average PCC for a large number of genes was obtained rather easily when only a small number of conditions were considered, a certain number of conditions should be required for each bicluster, to ensure the biological significance. The variation of $\alpha$ and $\beta$ was empirically estimated, and finally 0.3 and 0.5 were chosen, respectively (see the results in Table S1).

\section{Assessment procedure}

Six existing methods were compared to evaluate the performance of BIGA: Cheng and Church algorithm, ${ }^{9}$ StatisticalAlgorithmic Method for Bicluster Analysis (SAMBA), ${ }^{19,20}$ order-preserving submatrix (OPSM), ${ }^{1}$ iterative signature algorithm (ISA), ${ }^{11}$ binary inclusion-maximal biclustering algorithm (BIMAX), ${ }^{21}$ and SEBI. ${ }^{16} \mathrm{SEBI}$ is selected as a representative of the GA-based biclustering approaches, ${ }^{15,16}$ because SEBI adopts an outstanding system to reduce the redundancy of biclusters and performs iterative evolutionary searches like BIGA. The five other methods are based on greedy searches. Data provided by Gasch et $\mathrm{al}^{22}$ was used for the analyses of Saccharomyces cerevisiae. The analyses contained 2993 genes and 173 stress conditions, as a result the data size was large and abundant annotations were available. Prelic et $\mathrm{al}^{21}$ used this dataset to evaluate algorithms, and the resultant sets of biclusters for the five greedy-search algorithms are publicly available. These bicluster sets were obtained for comparison with our results. Neither the results of SEBI for the data nor SEBI itself is publicly available. The framework of SEBI was re-implemented in a second experiment. ${ }^{16}$ Note that there might be some minor differences between SEBI and the reimplemented SEBI. Henceforth, we denote mySEBI as our implementation.

The sets of biclusters were evaluated in terms of the following four points. Since PCC is a widely used parameter to assess the similarity of expression patterns, the distribution of the average PCC of all biclusters was examined. One may consider the mean square residual (MSR) of biclusters ${ }^{9}$ to be useful as an indicator of the coherence of biclusters, but PCC is better than MSR in terms of finding the functional relevance of genes, ${ }^{23-26}$ in much biological data, for example, the involvement of the same pathway or the participation in the same protein complex. ${ }^{27,28}$ The existing methods do not 
necessarily optimize the correlation of biclusters, and some biclusters derived from other algorithms can contain biclusters showing strong anti-correlation (ie, genes expressed inversely). The absolute value of PCC was used to estimate such biclusters for comparisons.

Coverage and overlap are also important measures to evaluate the biclustering, as higher coverage and lower overlap are preferable for further biological analyses. Previous studies ${ }^{29}$ used "cell coverage," by calculating the percentages of area (genes $\times$ conditions) covered by the biclusters, and "cell overlap" by measuring the intersection areas of the biclusters. In this study, "gene coverage" and "gene overlap," were adopted because higher cell coverage can be achieved even by a high coverage of conditions and a low coverage of genes, and this result is not biologically significant. In addition, cell overlap ignores the overlap of genes shared in any two biclusters, if the conditions in the biclusters are completely different. Gene coverage is defined as the ratio of genes that are assigned to any biclusters to all genes, and gene overlap is the ratio of total genes overlapping on multiple biclusters to the genes assigned to any biclusters (Equation 2):

$$
\text { Gene overlap }=\frac{\sum_{i=1}^{k} X_{i}-\left|\bigcup_{i=1}^{k} X_{i}\right|}{\left|\bigcup_{i=1}^{k} X_{i}\right|}
$$

Gene coverage can evaluate the ability of an algorithm to decide the cluster for each gene, and gene overlap can measure the ability of an algorithm to specify the clusters for genes that are not necessarily involved in multiple biological processes.

The biological significance of the results by measuring the GO enrichment was also evaluated. More precisely, FuncAssociate (2.0; Roth Laboratories, Harvard University, Boston, MA), a tool for finding overrepresented GO terms in a set of genes was utilised. Using this tool, we performed Fisher's exact test to determine the probability of the appearance of genes associated with a GO term in each bicluster. ${ }^{30}$ FuncAssociate calculates an adjusted $P$-value ( $P$ adj) from the simulations, instead of the corrections of multiple tests. Padj is the probability of obtaining at least one false positive for any desired cutoff. We considered a biologically significant bicluster as one that is relevant to at least one GO term with a statistically significant appearance (namely, Padj less than significance level). The number of such biclusters, relative to the total number of biclusters (the GO enrichment), was used to estimate each algorithm. A previous study by Prelic et $\mathrm{al}^{21}$ evaluated the biological relevance of existing algorithms, using the GO enrichment.

\section{Results and discussion Biclusters for the Saccharomyces cerevisiae microarray data}

With the selected parameters and thresholds, BIGA found 164 biclusters from the $S$. cerevisiae microarray data. The average numbers of genes and conditions in the biclusters are 92.25 and 23.65, respectively (Table 1). The detailed statistics of each bicluster are provided in Table S2. The properties of the biclusters obtained by other methods are also summarized in Table 1.

\section{Performance evaluation}

The distribution of the average PCCs of the biclusters obtained by each biclustering algorithm is shown in the boxplot (Figure 2A). The thick line around the middle of the box indicates the median of the average PCCs. The top and bottom of the box indicate the upper and the lower quartiles, respectively. The circles show the outliers (more than 1.5 times the upper quartile or less than 1.5 times the lower quartile from the median). The whiskers mean the range of data between the maximum and the minimum values, other than the outliers. According to the plots, OPSM performs the best with a very small deviation in the average PCCs. Apart from OPSM, BIGA can outperform the other methods when compared by the median of the average PCC. One may consider that the fitness function of BIGA takes the average PCC into account (Equation 1), and thus it is obvious that the average PCC of BIGA is good. However, note that the results are not necessarily satisfactory if the optimization procedure does not work well, or the balance between the average PCC and the area of the bicluster in (Equation 1) is inappropriate. Next, using the the Wilcoxon signed-rank test the study examined whether the distribution of the average PCCs of BIGA is significantly better than those of the other algorithms. ${ }^{31}$ The results showed that BIGA detects significantly more co-expressed genes in biclusters than the other methods, except for OPSM (the highest $P$-value is only $5.4 \times 10^{-6}$ against SAMBA). To clarify the performance, the expression profiles of the four best biclusters with higher average PCCs are demonstrated in Figure S1. Note: the reason for the highest performance of OPSM was related to the gene coverage and these analyses will be discussed later.

The gene coverage and the gene overlap are shown in (Figure 2B and 2C), respectively. As a result, BIGA achieved the fourth-highest gene coverage among the seven 
Table I Comparing quantitative metrics among biclustering algorithms

\begin{tabular}{llllllll}
\hline Properties & CC & SAMBA & ISA & OPSM & BIMAX & mySEBI & BIGA \\
\hline Number of biclusters & 100 & 100 & 66 & 12 & 101 & 100 & 164 \\
Average gene number & 82.01 & 911.52 & 76.27 & 95.58 & 24.03 & 74.98 & 92.25 \\
Average condition number & 19.85 & 25.15 & 8.71 & 12.50 & 3.00 & 80.5 & 23.65 \\
\hline
\end{tabular}

Abbreviations: BIGA, binary-iterative genetic algorithm; BIMAX, binary inclusion-maximal biclustering algorithm; CC, Cheng and Church algorithm; ISA, iterative signature algorithm; OPSM, order-preserving submatrix; mySEBI, the Sequential Evolutionary Biclustering method used in this work; SAMBA, Statistical-Algorithmic Method for Bicluster Analysis.
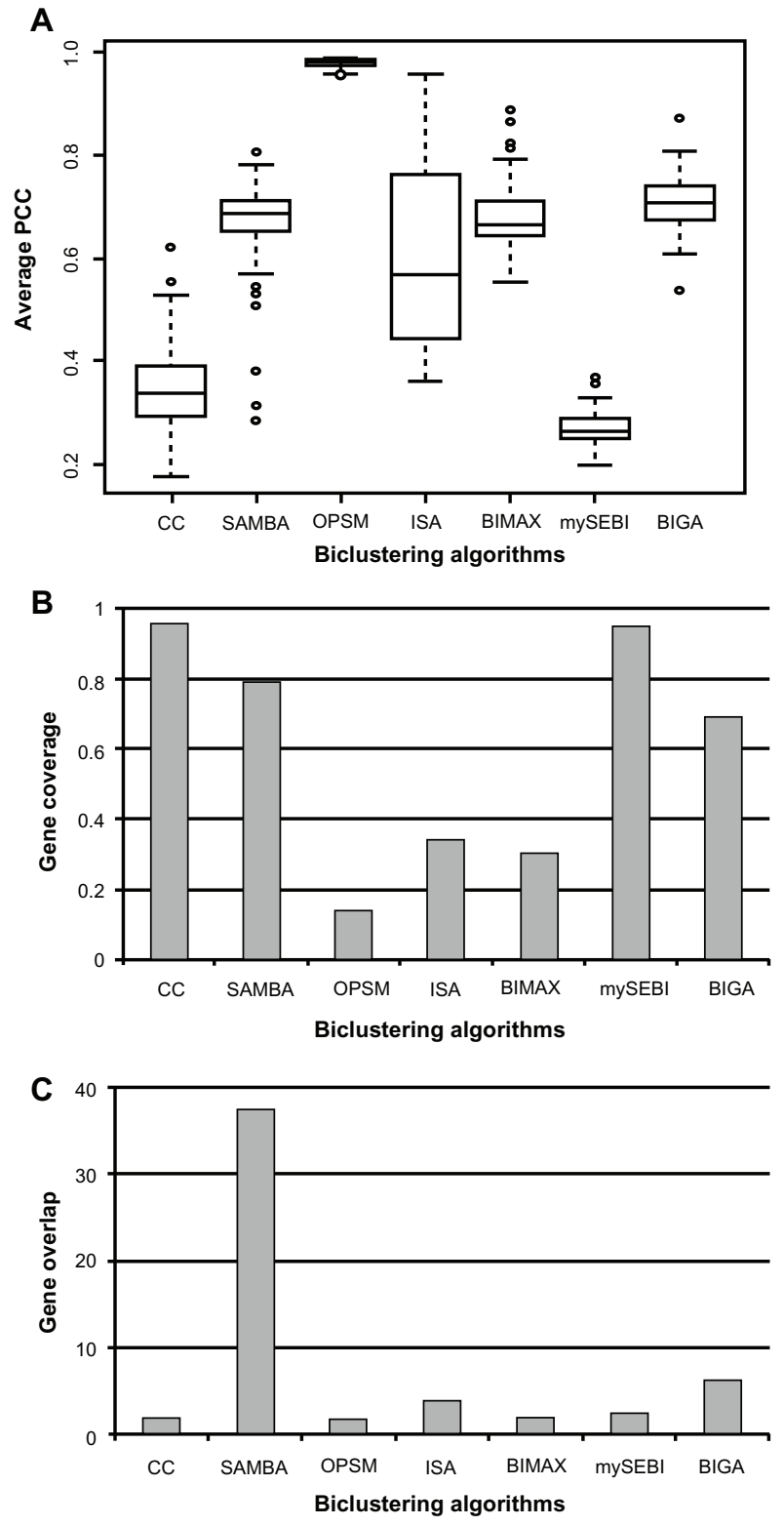

Figure 2 (A) Distribution of the average Pearson correlation coefficients for each biclustering algorithm, represented by a boxplot. (B) Histogram of gene coverage for each biclustering algorithm. The $y$-axis represents the coverage ratio between the union of genes appearing on biclusters and all analyzed genes. Higher coverage shows higher performance. (C) Histogram of gene overlap for each biclustering algorithm. The $y$-axis shows the gene overlap defined by (Equation 2). Lower overlap shows higher performance.

Abbreviations: CC, Cheng and Church algorithm; SAMBA, Statistical-Algorithmic Method for Bicluster Analysis; OPSM, order-preserving submatrix; ISA, iterative signature algorithm; BIMAX, binary inclusion-maximal biclustering algorithm; mySEBI, the Sequential Evolutionary Biclustering method used in this work; BIGA, binary-iterative genetic algorithm. algorithms (Figure 2B). SAMBA could classify almost 100\% of the genes into biclusters, but each bicluster contained more than 900 genes (Table 1) with extremely high overlap (Figure 2C), which will make the succeeding experimental or bioinformatics analyses difficult. mySEBI could produce a set of biclusters that would include $95 \%$ of all genes with a small amount of overlap. CC showed the best gene coverage (highest) and overlap (lowest). The results indicate that the techniques to reduce redundancy of biclusters in SEBI and $\mathrm{CC}$ are efficient for gaining high coverage and low overlap. However, the average PCCs of the biclusters by both algorithms were very low (Figure 2A). OPSM produced biclusters with the highest correlation (Figure 2A), but failed to achieve higher gene coverage due to the small number of clusters (Table 1). The average PCCs of OPSM and BIGA are high, because both methods adopt gene co-expression in the target function. By contrast, CC and SEBI adopt MSR instead of PCC. Although MSR can sometimes identify coherent biclusters, it is not necessarily efficient to achieve higher correlations of genes.

BIGA yielded the second-largest gene overlap, with 6.29 (Figure 2C), which may imply that the biclusters of BIGA are mutually similar. The pairwise overlap $(P O)$ of two biclusters defined by $X_{i} \cap X_{j} / X_{i} \cup X_{j}$, where $X_{i}$ and $X_{j}$ are genes in biclusters $B_{i}$ and $B_{j}$, respectively, was measured to examine the similarity of the biclusters more directly, and plotted in Figure 3A. The median of the POs for BIGA was not very large, as compared with those of the other methods, indicating that the biclusters determined by BIGA are not necessarily similar. Moreover, the variety of biclusters using the single-linkage clustering method, where the distance between two biclusters defined by $1.0-P O$ was investigated. At each cut-off distance, the number of clusters was counted and normalized by the total number of biclusters, which we call the fraction of independent biclusters. When the cut-off distance is sufficiently small, no biclusters are merged and $F I B$ is 1.0. This state indicates that the biclusters are independent and diverse. On the other hand, when the cut-off distance is sufficiently large, most of the biclusters may be merged together, and FIB will 


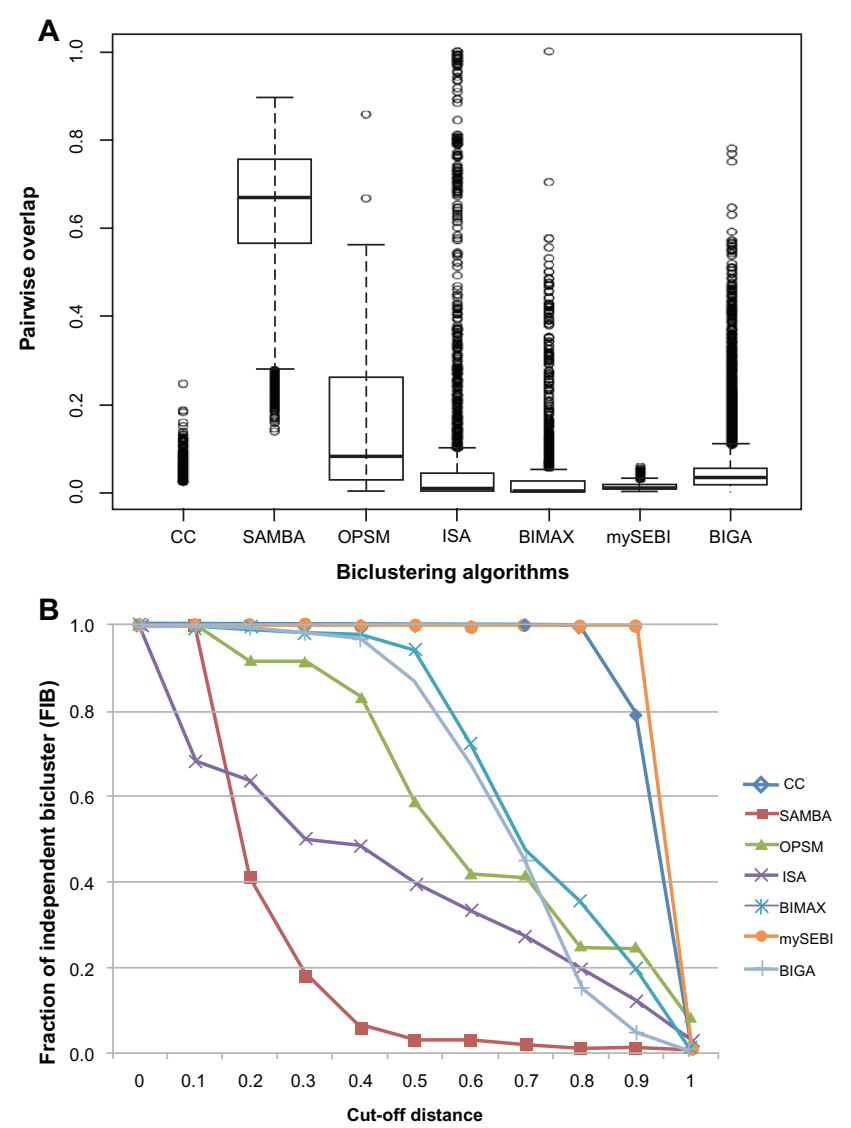

Figure 3 (A) Distribution of pairwise overlap $(P O)$ of biclusters, shown in boxplots for each algorithm. Thick lines, boxes, whiskers, and circles indicate the same things as in (Figure 2A). (B) The fraction of independent biclusters (FIB) over the cut-off distance.

Abbreviations: CC, Cheng and Church algorithm; SAMBA, Statistical-Algorithmic Method for Bicluster Analysis (SAMBA); OPSM, order-preserving submatrix; ISA, iterative signature algorithm; BIMAX, binary inclusion-maximal biclustering algorithm; mySEBI, the Sequential Evolutionary Biclustering method used in this work; BIGA, binary-iterative genetic algorithm.

converge to 0.0 . This state means that all of the biclusters are judged as being similar to each other. We consider a higher $F I B$ to be an indicator illustrating the variety of the resultant biclusters. According to the plot (Figure 3B), the $F I B$ s of SAMBA and ISA are obviously low in almost the whole cut-off distance range, showing that their biclusters are rather similar. The FIBs of OPSM show that its ability to detect diverse biclusters is moderate. CC, mySEBI, BIMAX, and BIGA provided a wider variety of biclusters than the other algorithms, when the cut-off distance was less than 0.5. In summary, the average bicluster determined by BIGA contains many genes that are shared with other biclusters (Figure 2C): however, when focusing on each pair of biclusters, a small number of genes are shared (Figure 3A). Consequently, the biclusters determined by BIGA seem to be independent (Figure 3B), and cover most of the genes efficiently (Figure 2B).

\section{Evaluation of biological relevance by gene ontology enrichment analyses}

In the study by Prelic et $\mathrm{al}^{21}$ on the evaluation of existing methods using GO enrichment, OPSM showed the best performance $(100 \%$ of the biclusters were significant at the 0.05 significance level). However, it only produced twelve biclusters (Table 1), and thus the gene coverage was the lowest (Figure 2B). Less than half of the biclusters produced by $\mathrm{CC}$ were judged to be significant, ${ }^{21}$ probably because $\mathrm{CC}$ cannot detect biclusters with a higher average PCC (Figure 2A). The percentages of significant biclusters from mySEBI are $93 \%, 81 \%, 69 \%$, and $42 \%$ for the 0.05 , $0.01,0.005$, and 0.001 , respectively. By contrast, $94.5 \%$ of the biclusters produced by BIGA were judged to be significant at the 0.05 significance level. This value was changed to $88.4 \%, 86.0 \%$, and $79.3 \%$ for the $0.01,0.005$, and 0.001 significance levels, respectively. The performance of BIGA is almost the same as those of BIMAX and ISA in $\mathrm{GO}$ enrichment, ${ }^{21}$ but BIGA outperforms them in the gene coverage (Figure 2B).

There was a functional relationship between the resultant biclusters by BIGA, based on the enriched GO terms at the 0.001 significance level. Among the $122 \mathrm{GO}$-enriched terms, ribosome-related terms (ribosome GO:0005840, ribosomal subunit GO:0033279, etc) are abundant in many biclusters (50 biclusters). This observation was consistent with the fact that $60 \%$ of transcription was devoted to ribosomal ribonucleic acid (RNA), ${ }^{32}$ because genes with higher expression levels tend to be clustered. Apart from the ribosomerelated terms, primary metabolic (GO:0044238), translation (GO:0006412), protein-related (GO:0044267, GO:0019538), macromolecule-related (GO:0009059, GO:0034645, GO:0044260, GO:0043170), and biopolymer-related (GO:0043283, GO:0034960, GO:0043284, GO:0034961) processes also frequently appeared in several biclusters. This indicated that the genes involved in these terms are primary or essential in many biological processes. Five GO terms that are most enriched at the 0.001 significance level for each bicluster five specific GO terms among them are shown in Table S2.

Furthermore, the novel aspects of the biclusters identified by BIGA were examined. For each bicluster defined by BIGA, the $P O$ against all biclusters identified by the other five methods was measured and the maximum $P O$ was derived (Table S2). The highest value of the maximum $P O s$ was at most 0.12 , indicating that the biclusters defined by BIGA are quite different from those determined by the other methods. To explore the relationships of the genes that were detected 
only by BIGA, on the study examined the biclusters of BIGA that were not similar to any of the other biclusters; that is, the biclusters with maximum pair-wise similarity scores $<0.05$. In bicluster 109 (the maximum $P O=0.039$ with bicluster 29 of $\mathrm{CC}$ ), 16 out of 86 genes are involved in a cellular nitrogen metabolic process (GO:0034641), eg, SAS3 (YBL052C), TEF2 (YBR118W), and SWD3 (YBR175W), are co-expressed under twelve conditions. In bicluster 118 (0.037 with bicluster 56 of CC), 26 out of 66 genes, eg, RRN6 (YBL014C), ORC2 (YBR060C), and PAF1 (YBR279W), are involved in an RNA metabolic process (GO:0016070). In bicluster 160 (0.037, bicluster 24 of ISA), 33 out of 74 genes, such as HEK2 (YBL032W), ROX3 (YBL093C), and SIF2 (YBR103W), are related to a nucleic acid metabolic process (GO:0090304). These results demonstrate that BIGA is useful to reveal the functional relevance underlying the biclusters. Furthermore, some genes belonged to the same bicluster, even though they lacked known co-functional evidence (see the biclusters in Table S2 without significant GO terms). These genes represent promising experimental targets that bridge biological processes exhibiting co-expression under specific conditions.

\section{Conclusion}

The development of biclustering algorithms has allowed biologists to start unraveling the underlying functional mechanisms in living organisms. We propose BIGA as an alternative biclustering technique, since it was designed to address the conventional problems of the pre-existing methods. Biclustering is obviously advantageous in accounting for the overlap state among clusters, but the suitable amount of overlap is still ambiguous and different algorithms often produce solutions with various degrees of overlap. We tried to develop a novel chromosome-encoding mode that explicitly defines the overlap between biclusters. BIGA revealed that the most frequently appearing genes express their functions in fundamental and essential biological processes, such as translation. A microarray often consists of relatively few conditions, with respect to a large number of genes. The weighting of genes and conditions diminishes the bias between the number of genes and conditions, which helps to eliminate unreliable results, such as biclusters with very few conditions. We also applied an alternative index, the average PCC, which impacts the biological meaning, rather than the MSR, to measure the goodness of a bicluster. The analysis of GO enrichment demonstrated that most of our biclusters were significant, with one or more enriched GO terms. When evaluated with the five pre-existing algorithms, BIGA performed well in most of the properties with good balance, although it did not show the best performance for all criteria. A pair-wise comparison of our biclusters with those obtained by the other algorithms revealed the novel aspects of the biclusters that are distinct from those of the other methods. Since biological systems are quite complicated, resulting in high-dimensional data, it is quite difficult to answer all biological questions with a single approach. For new discoveries, we recommend the application of several approaches, including BIGA.

\section{Acknowledgments}

We would like to thank the Human Genome Center for providing computational resources to analyze all of the data, as well as for a scholarship from the Ministry of Education, Culture, Sports, Science and Technology to Sawannee Sutheeworapong. We would like to acknowledge Prof Kenta Nakai for providing good facilities to Sawannee Sutheeworapong in the early stage of this work. We also thank Dr Takeshi Obayashi for useful discussions in the early stage of this work.

\section{Authors' contributions}

$\mathrm{SS}, \mathrm{KK}$, and MO contributed to the overall research and the manuscript preparation. $\mathrm{KK}, \mathrm{MO}$, and $\mathrm{HO}$ were responsible for the project direction and financial support.

\section{Disclosure}

The authors report no conflicts of interest in this work.

\section{References}

1. Ben-Dor A, Chor B, Karp R, Yakhini Z. Discovering local structure in gene expression data: the order-preserving submatrix problem. J Comput Biol. 2003;10:373-384.

2. Ma X, Salunga R, Tuggle T, et al. Gene expression profiles of human breast cancer progression. Proc Natl Acad Sci U S A. 2003;100: 5974-5979.

3. Yamane D, Zahoor MA, Mohamed YM, et al. Microarray analysis reveals distinct signaling pathways transcriptionally activated by infection with bovine viral diarrhea virus in different cell types. Virus Res. 2009;142(1-2):188-199.

4. Wang RS, Wang Y, Zhang XS, Chen L. Inferring transcriptional regulatory networks from high-throughput data. Bioinformatics. 2007;23(22):3056-3064.

5. Hartigan JA, Wong MA. A k-means clustering algorithm. Appl Stat. 1979;28:100-108.

6. Sokal RR, Michener CD. A statistical method for evaluating systematic relationships. Univ Kansas Sci Bull. 1958;38:1409-1438.

7. Madeira SC, Oliveira AL. Biclustering algorithms for biological data analysis: a survey. IEEE/ACM. Trans Computat Biol Bioinform. 2004;1(1):24-45.

8. Hartigan JA. Direct clustering of a data matrix. J Am Stat Assoc. 1972;67(337):123-129.

9. Cheng Y, Church GM. Biclustering of expression data. In: Proceedings of the Eighth International Conference on Intelligent Systems for Molecular Biology. ISMB 2000, San Diego, CA; August 19-12, 2000. AAAI Press; 2000:93-103. 
10. Murali TM, Kasif S. Extracting conserved gene expression motifs from gene expression data. Proceedings of the Pacific Symposium on Biocomputing. PSB 2003, Lihue, HI, January 3-7, 2003. 2003;8:77-88.

11. Bergmann S, Ihmels J, Barkai N. Iterative signature algorithm for the analysis of large-scale gene expression data. Phys Rev E Stat Nonlin Soft Matter Phys. 2003;67:031902.

12. Peeters R. The maximum edge biclique problem in NP-complete. Discrete Appl Math. 2003;131(3):651-654.

13. Merz P, Zell A. Genetic Algorithms and Grouping Problems. Philadelphia, PA: John Wiley \& Sons; 1998.

14. Bleuler S, Prelic A, Zitzler E. An EA framework for biclustering of gene expression data. Proceedings of Congress on Evolutionary Computation, Portland, OR, June 19-23. June 19-23, 2004;4: 166-173.

15. Chakraborty A, Maka H. Biclustering of gene expression data using genetic algorithm. Proceedings of the 2005 IEEE Symposium on Computational Intelligence in Bioinformatics and Computational Biology, 2005. CIBCB 2005. La Jolla, CA, November 14-14, 2005:1-8.

16. Divina F, Aguilar-ruiz JS. Biclustering of expression data with evolutionary computation. IEEE Trans Knowl Data Eng. 2006;18:590-602.

17. DonaleK. TheArtofComputer Programming, vol 1: Fundamental algorithms. 3rd ed Boston, MA: Addison-Wesley. 1997;Section 2.3:318-348.

18. Meffert K, Rotstan N. JGAP-Java Genetic Algorithms and Genetic Programming Package. Available from: http://jgap.sf.nt/. Accessed July 10, 2012.

19. Tanay A, Sharan R, Shamir R. Discovering statistically significant biclusters in gene expression data. Bioinformatics. 2002;18:S136-S144.

20. Tanay A, Sharan R, Kupiec M, Shamir R. Revealing modularity and organization in the yeast molecular network by integrated analysis of highly heterogeneous genomewide data. Proc Natl Acad Sci US A. 2003;102(9):2981-2986.

21. Prelic A, Bleuler S, Zimmermann P, et al. A systematic comparison and evaluation of biclustering methods for gene expression data. Bioinformatics. 2006;22(9):1122-1129.
22. Gasch AP, Spellman PT, Kao CM, et al. Genomic expression programs in the response of yeast cells to environmental changes. Mol Biol Cell. 2000;11:4241-4257.

23. Aguilar-Ruiz J. Shifting and scaling patterns from gene expression data. Bioinformatics. 2005;21:3840-3845.

24. Pontes B, Divina F, Giraldez R, Aguilar-Ruiz JS. Virtual error: a new measure for evolutionary biclustering. Evol Comput, Machine Learning and Data Mining in Bioinformatics. 2007;4447:217-226.

25. Teng L, Chan L. Discovering biclusters by iteratively sorting with weighted correlation coefficient in gene expression data. J Signal Process Syst. 2008;50(3):267-280.

26. Ayadi W, Elloumi M, Hao J. A biclustering algorithm based on a bicluster enumeration tree: application to DNA microarray data. Bio Data Min. 2009;2:9.

27. Dandekar T, Snel B, Huynen M, Bork P. Conservation of gene order: a fingerprint of proteins that physically interact. Trends Biochem Sci. 1998;23:324-328.

28. Eisen MB, Spellman PT, Brown PO, Botstein D. Cluster analysis and display of genome-wide expression patterns. Proc Natl Acad Sci USA. 1998;95:14863-14868.

29. Waltman P, Kacmarczyk T, Bate AR, et al. Multi-species integrative biclustering. Genome Biol. 2010;11:R96.

30. Berriz GF, King OD, Bryant B, Sander C, Roth FP. Characterizing gene sets with FuncAssociate. Bioinformatics. 2003;19:2502-2504.

31. Wilcoxon F. Individual comparisons by ranking methods. Biometrics Bull. 1945;1(6):80-83.

32. Warner JR. The economics of ribosome biosynthesis in yeast. Trends Biochem Sci. 1999;24:437-440. 


\section{Supplementary data}

Table SI Parameter determination

\begin{tabular}{|c|c|c|c|c|c|c|}
\hline & \multicolumn{6}{|c|}{ Goodness of biclusters } \\
\hline & $\overline{\text { Genes }}$ & Conditions & Correlation & Biclusters & Coverage & Overlap \\
\hline \multicolumn{7}{|l|}{$\bar{\alpha}$} \\
\hline 0.1 & 72.15 & 22.84 & 0.74 & 111 & 0.59 & 3.53 \\
\hline 0.3 & 92.25 & 23.65 & 0.71 & 164 & 0.69 & 6.29 \\
\hline 0.5 & 102.22 & 24.42 & 0.7 & 252 & 0.67 & 11.82 \\
\hline \multicolumn{7}{|l|}{$\tau_{r}$} \\
\hline 0.1 & 81.22 & 21.51 & 0.73 & 355 & 0.74 & 11.97 \\
\hline 0.15 & 92.25 & 23.65 & 0.71 & 164 & 0.69 & 6.29 \\
\hline 0.2 & 109.86 & 25.07 & 0.69 & 57 & 0.58 & 2.59 \\
\hline 0.25 & 128.13 & 32.5 & 0.71 & 8 & 0.22 & 0.53 \\
\hline 0.3 & 163 & 45 & 0.67 & I & 0.05 & 0 \\
\hline \multicolumn{7}{|l|}{$\tau_{c}$} \\
\hline 0.60 & 100.62 & 22.17 & 0.69 & 145 & 0.71 & 5.9 \\
\hline 0.65 & 92.25 & 23.65 & 0.71 & 164 & 0.69 & 6.29 \\
\hline 0.70 & 83.84 & 22.69 & 0.74 & 178 & 0.61 & 7.09 \\
\hline
\end{tabular}

Notes: (A) Impact of gene-weight parameter on the goodness of biclusters $(\tau=30, \tau=10, \tau=0.65, \tau=0.15$ and $\beta=0.5)$. (B) Impact of redundant threshold on the goodness of biclusters $\left(\tau_{n}=30, \tau_{m}=10, \tau_{c}=0.65\right.$, and $\left.\alpha=0.3, \beta=0.5\right)$. (C) Impact of correlation threshold on the goodness of biclusters $\left(\tau_{n}=30, \tau_{m}=10, \tau_{c}=0.15\right.$, and $\alpha=0.3, \beta=0.5)$
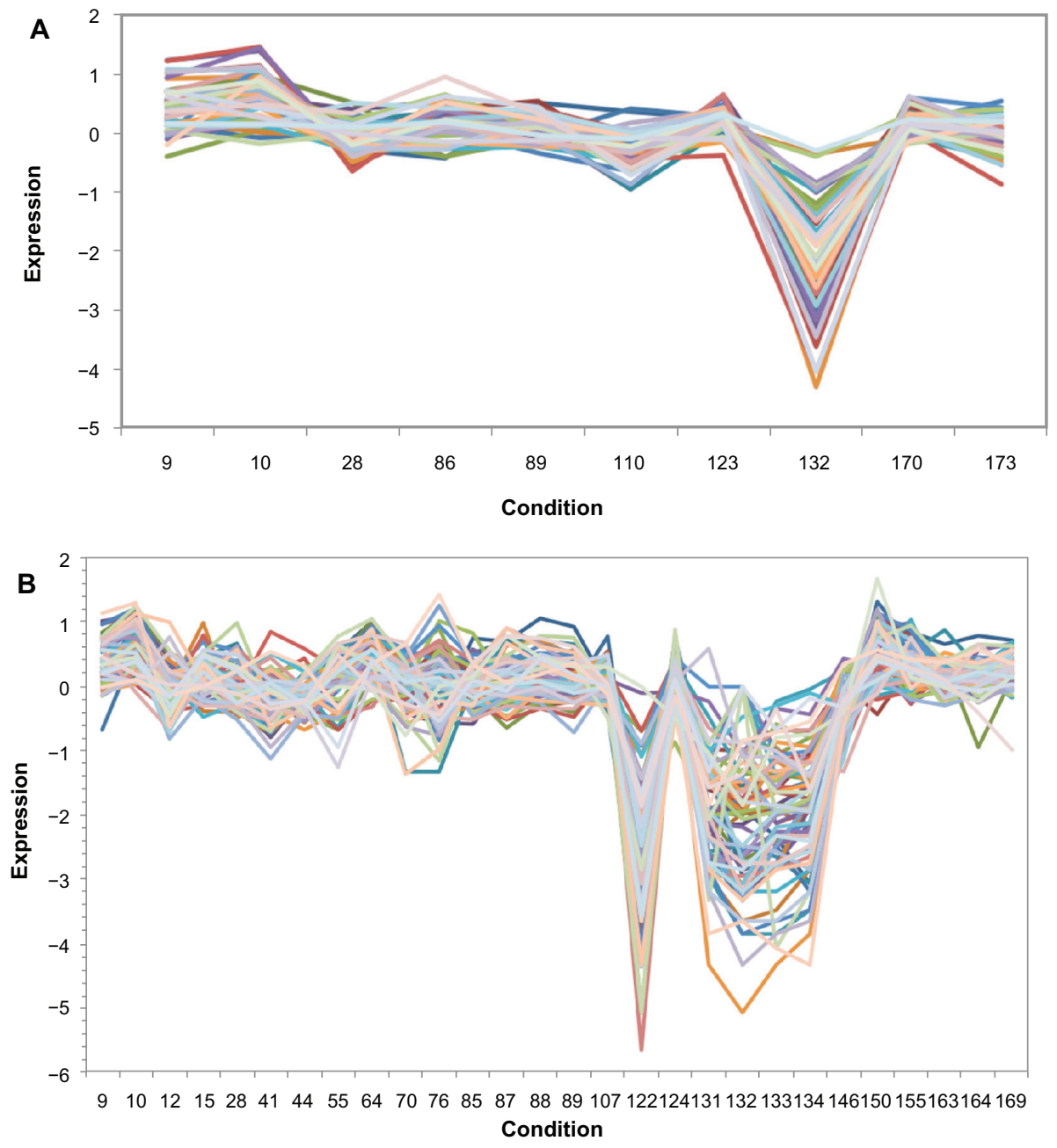

Figure SI (Continued) 

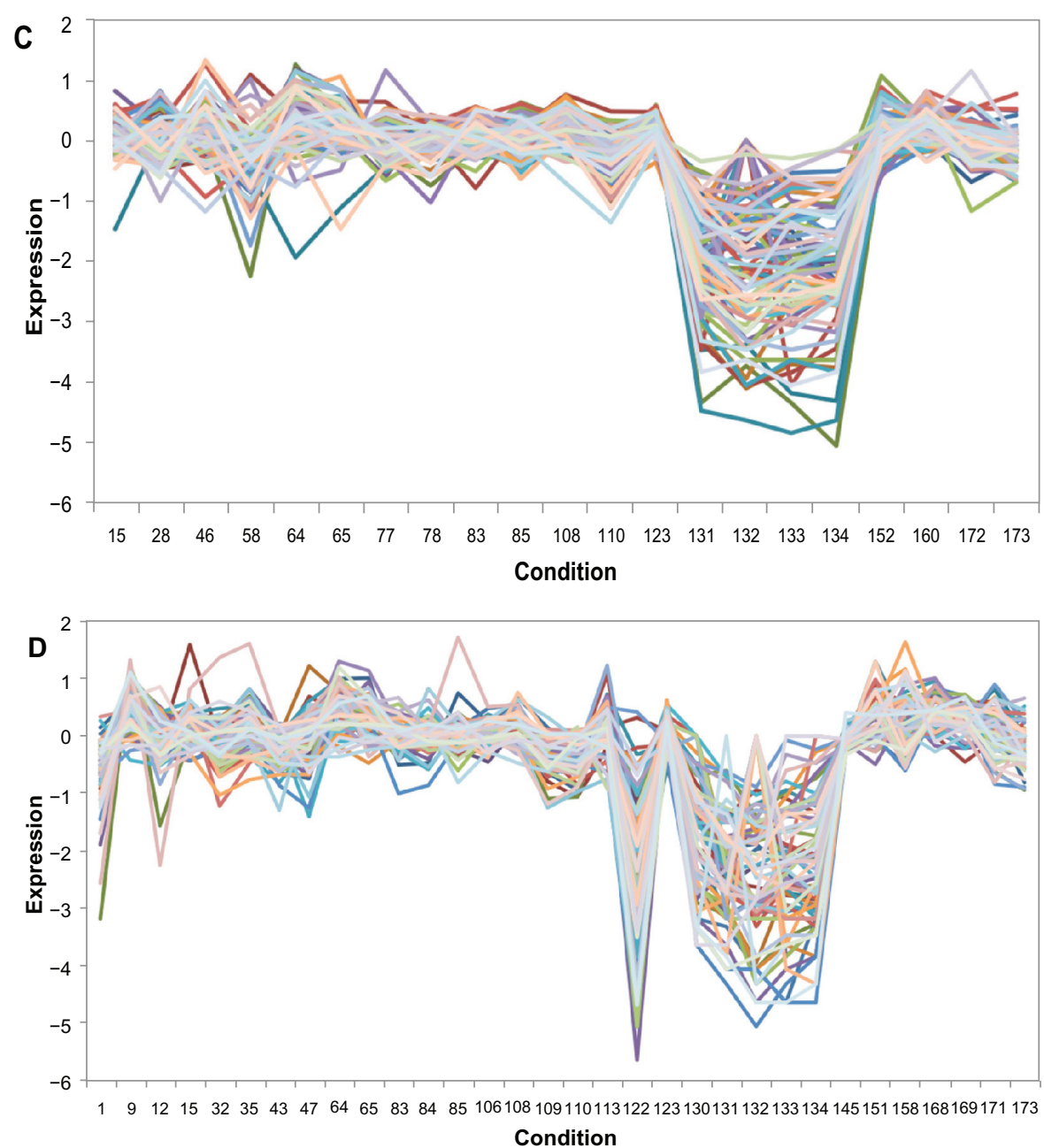

Figure SI Expression profiles of biclusters I (A), 2 (B), 3 (C), and 4 (D), in the descending order of the average Pearson's correlation coefficient. Note: The $x$-axis represents the series of conditions; eg, the number 8 denotes the 8 th condition. 
Table S2 Detailed statistics of resulting biclusters (sorted by descending order of average PCC)

\begin{tabular}{|c|c|c|c|c|c|}
\hline Bicluster ID & $\begin{array}{l}\text { Number } \\
\text { of genes }\end{array}$ & $\begin{array}{l}\text { Number of } \\
\text { conditions }\end{array}$ & $\begin{array}{l}\text { Average } \\
\text { PCC }\end{array}$ & $\begin{array}{l}\text { The minimum adjusted } \\
P \text {-value of } G O \text { enrichment }\end{array}$ & $\begin{array}{l}\text { Number of enriched } \\
\text { GO terms }\end{array}$ \\
\hline I & 47 & 10 & 0.87 & $<0.001$ & 2 \\
\hline 2 & 74 & 28 & 0.81 & $<0.001$ & 3 \\
\hline 3 & 85 & 21 & 0.80 & $<0.001$ & 14 \\
\hline 4 & 71 & 32 & 0.80 & & 12 \\
\hline 5 & 74 & 18 & 0.80 & 0.001 & I \\
\hline 6 & 50 & 7 & 0.80 & - & 0 \\
\hline 7 & 79 & 24 & 0.80 & $<0.001$ & 8 \\
\hline
\end{tabular}

16

4

21

20

26

14

33

23

18
0.79

0.79

0.79

0.79

$<0.00$ I

$<0.001$

$\begin{array}{ll}0.79 & <0.001 \\ 0.78 & <0.001\end{array}$

$\begin{array}{ll}0.79 & <0.001 \\ 0.78 & <0.001\end{array}$

0.78

$<0.00$ l

2

0.78

$<0.001$

10 


\begin{tabular}{|c|c|c|}
\hline Five most significant GO terms & Five most specific GO terms & $\begin{array}{l}\text { Highest pairwise } \\
\text { simirarity score }\end{array}$ \\
\hline $\begin{array}{l}\text { GO:0003674 molecular_function } \\
\text { GO:003299I macromolecular complex }\end{array}$ & - & 0.044 \\
\hline GO:0003674 molecular_function & - & 0.067 \\
\hline \multicolumn{3}{|l|}{ GO:003299I macromolecular complex } \\
\hline \multicolumn{3}{|l|}{ GO:0043234 protein complex } \\
\hline GO:0043228 nonmembrane-bounded organelle & GO:0007II 4 cell budding & 0.070 \\
\hline GO:0043232 intracellular nonmembrane-bounded & GO:0022618 ribonucleoprotein complex assembly & \\
\hline organelle & GO:0032505 reproduction of a single-celled organism & \\
\hline GO:0044238 primary metabolic process & GO:0042257 ribosomal subunit assembly & \\
\hline GO:003299I macromolecular complex & GO:0043933 macromolecular complex subunit organization & \\
\hline \multicolumn{3}{|l|}{ GO:0022618 ribonucleoprotein complex assembly } \\
\hline GO:0030529 ribonucleoprotein complex & GO:0022625 cytosolic large ribosomal subunit & 0.093 \\
\hline \multicolumn{3}{|l|}{ GO:003299I macromolecular complex } \\
\hline \multicolumn{3}{|l|}{ GO:0005840 ribosome } \\
\hline \multicolumn{3}{|l|}{ GO:0044445 cytosolic part } \\
\hline \multicolumn{3}{|l|}{ GO:00064I 2 translation } \\
\hline GO:0005737 cytoplasm & GO:0005737 cytoplasm & 0.050 \\
\hline- & - & 0.043 \\
\hline GO:0044238 primary metabolic process & GO:0009072 aromatic amino acid family metabolic process & 0.073 \\
\hline \multicolumn{3}{|l|}{ GO:003299I macromolecular complex } \\
\hline \multicolumn{3}{|l|}{ GO:0043228 nonmembrane-bounded organelle } \\
\hline \multirow{2}{*}{\multicolumn{3}{|c|}{$\begin{array}{l}\text { GO:0043232 intracellular nonmembrane-bounded } \\
\text { organelle }\end{array}$}} \\
\hline & & \\
\hline \multicolumn{3}{|l|}{ GO:0005840 ribosome } \\
\hline- & - & 0.032 \\
\hline- & - & 0.041 \\
\hline GO:0003674 molecular_function & GO:0044249 cellular biosynthetic process & 0.068 \\
\hline GO:00064I 2 translation & GO:0009058 biosynthetic process & \\
\hline \multicolumn{3}{|l|}{ GO:0009987 cellular process } \\
\hline \multicolumn{3}{|l|}{ GO:0009058 biosynthetic process } \\
\hline \multicolumn{3}{|l|}{ GO:0044249 cellular biosynthetic process } \\
\hline GO:003299I macromolecular complex & - & 0.060 \\
\hline \multicolumn{3}{|l|}{ GO:0003674 molecular_function } \\
\hline \multicolumn{3}{|l|}{ GO:0009987 cellular process } \\
\hline \multicolumn{3}{|l|}{ GO:0043228 nonmembrane-bounded organelle } \\
\hline \multicolumn{3}{|l|}{ GO:0043232 intracellular nonmembrane-bounded } \\
\hline \multicolumn{3}{|l|}{ organelle } \\
\hline GO:0032040 small-subunit processome & GO:0032040 small-subunit processome & 0.074 \\
\hline GO:0030686 90S preribosome & GO:0022613 ribonucleoprotein complex biogenesis & \\
\hline GO:0042254 ribosome biogenesis & GO:0042254 ribosome biogenesis & \\
\hline GO:0030684 preribosome & GO:0030684 preribosome & \\
\hline GO:0022613 ribonucleoprotein complex biogenesis & GO:0030686 90 S preribosome & \\
\hline GO:0003674 molecular_function & - & 0.048 \\
\hline GO:0044445 cytosolic part & GO:00I5934 large ribosomal subunit & 0.080 \\
\hline GO:00064I 2 translation & GO:0022625 cytosolic large ribosomal subunit & \\
\hline GO:0022625 cytosolic large ribosomal subunit & GO:0044249 cellular biosynthetic process & \\
\hline GO:0043228 nonmembrane-bounded organelle & GO:0009058 biosynthetic process & \\
\hline GO:0043232 intracellular nonmembrane-bounded & & \\
\hline organelle & & \\
\hline GO:0003674 molecular_function & - & 0.056 \\
\hline GO:003299I macromolecular complex & & \\
\hline GO:0044238 primary metabolic process & GO:0008152 metabolic process & 0.059 \\
\hline GO:00I6070 RNA metabolic process & GO:00I6070 RNA metabolic process & \\
\hline GO:0044260 cellular macromolecule & GO:0034960 cellular biopolymer metabolic process & \\
\hline metabolic process & GO:0044260 cellular macromolecule metabolic process & \\
\hline GO:0043283 biopolymer metabolic process & GO:0044237 cellular metabolic process & \\
\hline GO:0030529 ribonucleoprotein complex & & \\
\hline
\end{tabular}


Table S2 (Continued)

\begin{tabular}{llllll}
\hline Bicluster ID & $\begin{array}{l}\text { Number } \\
\text { of genes }\end{array}$ & $\begin{array}{l}\text { Number of } \\
\text { conditions }\end{array}$ & $\begin{array}{l}\text { Average } \\
\text { PCC }\end{array}$ & $\begin{array}{l}\text { The minimum adjusted } \\
\text { P-value of GO enrichment }\end{array}$ & $\begin{array}{l}\text { Number of enriched } \\
\text { GO terms }\end{array}$ \\
\hline 17 & 92 & 23 & 0.78 & $<0.001$ & 12
\end{tabular}




\begin{tabular}{|c|c|c|}
\hline Five most significant GO terms & Five most specific GO terms & $\begin{array}{l}\text { Highest pairwise } \\
\text { simirarity score }\end{array}$ \\
\hline GO:0044238 primary metabolic process & GO:003462I cellular macromolecular complex & 0.072 \\
\hline GO:003299I macromolecular complex & subunit organization & \\
\hline GO:0043228 nonmembrane-bounded organelle & GO:0034660 ncRNA metabolic process & \\
\hline GO:0043232 intracellular nonmembrane- & GO:0006139 "nucleobase, nucleoside, nucleotide & \\
\hline bounded organelle & and nucleic acid metabolic process" & \\
\hline GO:003462I cellular macromolecular & GO:00I6070 RNA metabolic process & \\
\hline complex subunit organization & GO:0044237 cellular metabolic process & \\
\hline GO:0003674 molecular_function & - & 0.050 \\
\hline \multicolumn{3}{|l|}{ GO:0044445 cytosolic part } \\
\hline \multicolumn{3}{|l|}{ GO:0009987 cellular process } \\
\hline \multicolumn{3}{|l|}{ GO:003299I macromolecular complex } \\
\hline GO:0003674 molecular_function & GO:00I5935 small ribosomal subunit & 0.062 \\
\hline \multicolumn{3}{|l|}{ GO:003299I macromolecular complex } \\
\hline \multicolumn{3}{|l|}{ GO:0044238 primary metabolic process } \\
\hline \multicolumn{3}{|l|}{ GO:0030529 ribonucleoprotein complex } \\
\hline \multicolumn{3}{|l|}{ GO:0015935 small ribosomal subunit } \\
\hline GO:0044238 primary metabolic process & - & 0.046 \\
\hline GO:0043228 nonmembrane-bounded organelle & GO:0005737 cytoplasm & 0.073 \\
\hline \multicolumn{3}{|l|}{ GO:0043232 intracellular nonmembrane- } \\
\hline \multicolumn{3}{|l|}{ bounded organelle } \\
\hline \multicolumn{3}{|l|}{ GO:003299I macromolecular complex } \\
\hline \multicolumn{3}{|l|}{ GO:0044445 cytosolic part } \\
\hline \multicolumn{3}{|l|}{ GO:0005840 ribosome } \\
\hline GO:0044238 primary metabolic process & - & 0.058 \\
\hline GO:003299I macromolecular complex & GO:005 I 246 regulation of protein metabolic process & 0.059 \\
\hline GO:0043283 biopolymer metabolic process & GO:0034960 cellular biopolymer metabolic process & \\
\hline GO:0034960 cellular biopolymer metabolic & GO:0044260 cellular macromolecule metabolic process & \\
\hline process & GO:0032268 regulation of cellular protein metabolic process & \\
\hline GO:0043234 protein complex & GO:0043234 protein complex & \\
\hline \multicolumn{3}{|l|}{ GO:0043 I 70 macromolecule metabolic process } \\
\hline- & - & 0.039 \\
\hline GO:0003674 molecular_function & - & 0.045 \\
\hline GO:0044238 primary metabolic process & GO:0003743 translation initiation factor activity & 0.077 \\
\hline GO:0003674 molecular_function & GO:0045 I 82 translation regulator activity & \\
\hline GO:0009987 cellular process & GO:0008I35 "translation factor activity, nucleic acid binding" & \\
\hline GO:0005840 ribosome & GO:0032268 regulation of cellular protein metabolic process & \\
\hline GO:0003735 structural constituent of ribosome & GO:0043234 protein complex & \\
\hline \multicolumn{3}{|l|}{ GO:0045I82 translation regulator activity } \\
\hline GO:0044238 primary metabolic process & GO:00I5935 small ribosomal subunit & 0.098 \\
\hline GO:0003735 structural constituent of ribosome & GO:0008I52 metabolic process & \\
\hline GO:0009987 cellular process & GO:0043229 intracellular organelle & \\
\hline GO:0005840 ribosome & GO:0043226 organelle & \\
\hline GO:0003735 structural constituent of ribosome & GO:0022627 cytosolic small ribosomal subunit & \\
\hline GO:0003674 molecular_function & - & 0.045 \\
\hline GO:0003674 molecular_function & - & 0.077 \\
\hline \multicolumn{3}{|l|}{ GO:003299I macromolecular complex } \\
\hline GO:0009058 biosynthetic process & GO:0044249 cellular biosynthetic process & 0.056 \\
\hline GO:0044249 cellular biosynthetic process & GO:0009058 biosynthetic process & \\
\hline \multicolumn{3}{|l|}{ GO:0044238 primary metabolic process } \\
\hline \multicolumn{3}{|l|}{ GO:003299I macromolecular complex } \\
\hline \multicolumn{3}{|l|}{ GO:0044445 cytosolic part } \\
\hline GO:0009058 biosynthetic process & GO:0006|39 “nucleobase, nucleoside, nucleotide & 0.063 \\
\hline GO:0044249 cellular biosynthetic process & and nucleic acid metabolic process" & \\
\hline GO:0043284 biopolymer biosynthetic process & GO:003496 I cellular biopolymer biosynthetic process & \\
\hline GO:0009059 macromolecule biosynthetic process & GO:0034645 cellular macromolecule biosynthetic process & \\
\hline \multirow[t]{2}{*}{ GO:0044238 primary metabolic process } & GO:00I6070 RNA metabolic process & \\
\hline & GO:0009059 macromolecule biosynthetic process & \\
\hline
\end{tabular}


Table S2 (Continued)

\begin{tabular}{llllll}
\hline Bicluster ID & $\begin{array}{l}\text { Number } \\
\text { of genes }\end{array}$ & $\begin{array}{l}\text { Number of } \\
\text { conditions }\end{array}$ & $\begin{array}{l}\text { Average } \\
\text { PCC }\end{array}$ & $\begin{array}{l}\text { The minimum adjusted } \\
\text { P-value of GO enrichment }\end{array}$ & $\begin{array}{l}\text { Number of enriched } \\
\text { GO terms }\end{array}$ \\
\hline 32 & 91 & 30 & 0.76 & $<0.001$ & 10
\end{tabular}




\begin{tabular}{|c|c|c|}
\hline Five most significant GO terms & Five most specific GO terms & $\begin{array}{l}\text { Highest pairwise } \\
\text { simirarity score }\end{array}$ \\
\hline GO:00I7III nucleoside-triphosphatase activity & GO:00I7III nucleoside-triphosphatase activity & 0.081 \\
\hline GO:00I6462 pyrophosphatase activity & GO:00I6462 pyrophosphatase activity & \\
\hline GO:00168I7 "hydrolase activity, acting & GO:00I68I7 "hydrolase activity, acting on acid anhydrides" & \\
\hline on acid anhydrides" & GO:00168I8 "hydrolase activity, acting on acid anhydrides, & \\
\hline GO:00168I8 "hydrolase activity, acting on acid & in phosphorus-containing anhydrides" & \\
\hline anhydrides, in phosphorus-containing anhydrides" & GO:0034470 ncRNA processing & \\
\hline \multicolumn{3}{|l|}{ GO:0044238 primary metabolic process } \\
\hline GO:0009058 biosynthetic process & GO:0009058 biosynthetic process & 0.098 \\
\hline \multicolumn{3}{|l|}{ GO:003299I macromolecular complex } \\
\hline \multicolumn{3}{|l|}{ GO:0009987 cellular process } \\
\hline \multicolumn{3}{|l|}{ GO:00064I 2 translation } \\
\hline \multicolumn{3}{|l|}{ GO:0044445 cytosolic part } \\
\hline GO:003299I macromolecular complex & GO:0044444 cytoplasmic part & 0.088 \\
\hline GO:0044267 cellular protein metabolic process & GO:0044424 intracellular part & \\
\hline GO:00064I 2 translation & GO:0043234 protein complex & \\
\hline GO:0009987 cellular process & GO:0009058 biosynthetic process & \\
\hline \multicolumn{3}{|l|}{ GO:0043234 protein complex } \\
\hline GO:003299I macromolecular complex & GO:00I9438 aromatic compound biosynthetic process & 0.085 \\
\hline GO:0016070 RNA metabolic process & GO:0006396 RNA processing & \\
\hline GO:0044238 primary metabolic process & GO:0034470 ncRNA processing & \\
\hline GO:0009987 cellular process & GO:0034660 ncRNA metabolic process & \\
\hline \multirow[t]{2}{*}{ GO:0005I 98 structural molecule activity } & GO:0006I39 "nucleobase, nucleoside, nucleotide & \\
\hline & and nucleic acid metabolic process" & \\
\hline GO:003299I macromolecular complex & GO:0022627 cytosolic small ribosomal subunit & 0.069 \\
\hline \multicolumn{3}{|l|}{ GO:0003735 structural constituent of ribosome } \\
\hline \multicolumn{3}{|l|}{ GO:0033279 ribosomal subunit } \\
\hline \multicolumn{3}{|l|}{ GO:0005I 98 structural molecule activity } \\
\hline \multicolumn{3}{|l|}{ GO:00064I 2 translation } \\
\hline GO:0044085 cellular component biogenesis & GO:0044085 cellular component biogenesis & 0.068 \\
\hline GO:0003674 molecular_function & - & 0.040 \\
\hline \multicolumn{3}{|l|}{ GO:0005198 structural molecule activity } \\
\hline \multicolumn{3}{|l|}{ GO:003299I macromolecular complex } \\
\hline GO:0044249 cellular biosynthetic process & GO:0000462 "maturation of SSU-rRNA from tricistronic & 0.048 \\
\hline GO:0043228 nonmembrane-bounded organelle & rRNA transcript (SSU-rRNA, 5.8S rRNA, LSU-rRNA)" & \\
\hline GO:0043232 intracellular nonmembrane- & GO:0030490 maturation of SSU-rRNA & \\
\hline bounded organelle & GO:003496 I cellular biopolymer biosynthetic process & \\
\hline GO:0009058 biosynthetic process & GO:0034645 cellular macromolecule biosynthetic process & \\
\hline GO:0043284 biopolymer biosynthetic process & GO:0022627 cytosolic small ribosomal subunit & \\
\hline GO:0044445 cytosolic part & GO:0043229 intracellular organelle & 0.076 \\
\hline GO:00064I 2 translation & GO:0043226 organelle & \\
\hline \multicolumn{3}{|l|}{ GO:0043229 intracellular organelle } \\
\hline \multicolumn{3}{|l|}{ GO:0043226 organelle } \\
\hline \multicolumn{3}{|l|}{ GO:0043228 nonmembrane-bounded organelle } \\
\hline GO:003299I macromolecular complex & GO:0043234 protein complex & 0.069 \\
\hline \multicolumn{3}{|l|}{ GO:0043234 protein complex } \\
\hline \multicolumn{3}{|l|}{ GO:0003674 molecular_function } \\
\hline \multicolumn{3}{|l|}{ GO:0044238 primary metabolic process } \\
\hline \multicolumn{3}{|l|}{ GO:0009987 cellular process } \\
\hline GO:0044445 cytosolic part & GO:0006913 nucleocytoplasmic transport & 0.080 \\
\hline GO:0030529 ribonucleoprotein complex & GO:005 II69 nuclear transport & \\
\hline GO:0005I 98 structural molecule activity & GO:0005622 intracellular & \\
\hline GO:0033279 ribosomal subunit & GO:0005737 cytoplasm & \\
\hline GO:00064I 2 translation & GO:0010608 posttranscriptional regulation of gene expression & \\
\hline
\end{tabular}


Table S2 (Continued)

\begin{tabular}{llllll}
\hline Bicluster ID & $\begin{array}{l}\text { Number } \\
\text { of genes }\end{array}$ & $\begin{array}{l}\text { Number of } \\
\text { conditions }\end{array}$ & $\begin{array}{l}\text { Average } \\
\text { PCC }\end{array}$ & $\begin{array}{l}\text { The minimum adjusted } \\
\text { P-value of GO enrichment }\end{array}$ & $\begin{array}{l}\text { Number of enriched } \\
\text { GO terms }\end{array}$ \\
\hline 43 & 90 & 22 & 0.74 & $<0.001$ & 18
\end{tabular}




\begin{tabular}{|c|c|c|}
\hline Five most significant GO terms & Five most specific GO terms & $\begin{array}{l}\text { Highest pairwise } \\
\text { simirarity score }\end{array}$ \\
\hline GO:003299I macromolecular complex & GO:0044249 cellular biosynthetic process & 0.081 \\
\hline GO:0022627 cytosolic small ribosomal subunit & GO:0009058 biosynthetic process & \\
\hline \multicolumn{3}{|l|}{ GO:0030684 preribosome } \\
\hline \multicolumn{3}{|l|}{ GO:0030686 90S preribosome } \\
\hline \multicolumn{3}{|l|}{ GO:0030529 ribonucleoprotein complex } \\
\hline GO:0044238 primary metabolic process & GO:003462I cellular macromolecular complex & 0.061 \\
\hline GO:003299I macromolecular complex & subunit organization & \\
\hline GO:0009987 cellular process & GO:0016070 RNA metabolic process & \\
\hline \multicolumn{3}{|l|}{ GO:003462 I cellular macromolecular } \\
\hline \multicolumn{3}{|l|}{ complex subunit organization } \\
\hline \multicolumn{3}{|l|}{ GO:00I6070 RNA metabolic process } \\
\hline GO:0019538 protein metabolic process & GO:0005737 cytoplasm & 0.057 \\
\hline GO:0044267 cellular protein metabolic process & GO:0010608 posttranscriptional regulation of gene expression & \\
\hline GO:0032268 regulation of cellular protein & GO:005 I 246 regulation of protein metabolic process & \\
\hline metabolic process & GO:0006417 regulation of translation & \\
\hline GO:0005737 cytoplasm & GO:0032268 regulation of cellular protein metabolic process & \\
\hline \multicolumn{3}{|l|}{ GO:005 I 246 regulation of protein metabolic process } \\
\hline GO:0009987 cellular process & GO:0022627 cytosolic small ribosomal subunit & 0.089 \\
\hline \multicolumn{3}{|l|}{ GO:00064I 2 translation } \\
\hline \multicolumn{3}{|l|}{ GO:003299I macromolecular complex } \\
\hline \multicolumn{3}{|l|}{ GO:0044445 cytosolic part } \\
\hline \multicolumn{3}{|l|}{ GO:0044238 primary metabolic process } \\
\hline GO:0030529 ribonucleoprotein complex & GO:0016462 pyrophosphatase activity & 0.100 \\
\hline GO:0043228 nonmembrane-bounded organelle & GO:0016817 "hydrolase activity, acting on acid anhydrides" & \\
\hline GO:0043232 intracellular nonmembrane- & GO:0016818 "hydrolase activity, acting on acid anhydrides, & \\
\hline bounded organelle & in phosphorus-containing anhydrides" & \\
\hline \multicolumn{3}{|l|}{ GO:0005840 ribosome } \\
\hline \multicolumn{3}{|l|}{ GO:003299I macromolecular complex } \\
\hline GO:003299I macromolecular complex & GO:0005622 intracellular & 0.083 \\
\hline GO:0044238 primary metabolic process & GO:0022625 cytosolic large ribosomal subunit & \\
\hline GO:0044445 cytosolic part & GO:0010608 posttranscriptional regulation of gene expression & \\
\hline GO:0009987 cellular process & GO:005 I 246 regulation of protein metabolic process & \\
\hline GO:0005840 ribosome & GO:00064I7 regulation of translation & \\
\hline GO:003299I macromolecular complex & GO:003496I cellular biopolymer biosynthetic process & 0.082 \\
\hline GO:0044445 cytosolic part & GO:0034645 cellular macromolecule biosynthetic process & \\
\hline GO:0005840 ribosome & GO:0022627 cytosolic small ribosomal subunit & \\
\hline GO:0005I 98 structural molecule activity & GO:0034960 cellular biopolymer metabolic process & \\
\hline GO:00064I 2 translation & GO:0009059 macromolecule biosynthetic process & \\
\hline GO:0030529 ribonucleoprotein complex & GO:0005488 binding & 0.074 \\
\hline \multicolumn{3}{|l|}{ GO:003299I macromolecular complex } \\
\hline \multicolumn{3}{|l|}{ GO:0044238 primary metabolic process } \\
\hline \multicolumn{3}{|l|}{ GO:0005840 ribosome } \\
\hline \multicolumn{3}{|l|}{ GO:0043228 nonmembrane-bounded organelle } \\
\hline GO:0003674 molecular_function & GO:0000I66 nucleotide binding & 0.065 \\
\hline \multicolumn{3}{|l|}{ GO:0009987 cellular process } \\
\hline \multicolumn{3}{|l|}{ GO:0000I66 nucleotide binding } \\
\hline GO:00064I 2 translation & GO:0006082 organic acid metabolic process & 0.119 \\
\hline GO:003299I macromolecular complex & GO:00I 9752 carboxylic acid metabolic process & \\
\hline GO:0009058 biosynthetic process & GO:0005737 cytoplasm & \\
\hline GO:0009987 cellular process & GO:0009059 macromolecule biosynthetic process & \\
\hline GO:0044249 cellular biosynthetic process & GO:0043284 biopolymer biosynthetic process & \\
\hline GO:003299I macromolecular complex & GO:0007010 cytoskeleton organization & 0.062 \\
\hline GO:0044445 cytosolic part & GO:00I5935 small ribosomal subunit & \\
\hline GO:0043228 nonmembrane-bounded organelle & GO:0022627 cytosolic small ribosomal subunit & \\
\hline GO:0043232 intracellular nonmembrane- & GO:0006417 regulation of translation & \\
\hline bounded organelle & GO:0032268 regulation of cellular protein metabolic process & \\
\hline GO:0005I 98 structural molecule activity & & \\
\hline
\end{tabular}


Table S2 (Continued)

\begin{tabular}{llllll}
\hline Bicluster ID & $\begin{array}{l}\text { Number } \\
\text { of genes }\end{array}$ & $\begin{array}{l}\text { Number of } \\
\text { conditions }\end{array}$ & $\begin{array}{l}\text { Average } \\
\text { PCC }\end{array}$ & $\begin{array}{l}\text { The minimum adjusted } \\
\text { P-value of GO enrichment }\end{array}$ & $\begin{array}{l}\text { Number of enriched } \\
\text { GO terms }\end{array}$ \\
\hline 54 & 68 & 24 & 0.73 & 0.001 & 6
\end{tabular}




\begin{tabular}{|c|c|c|}
\hline Five most significant GO terms & Five most specific GO terms & $\begin{array}{l}\text { Highest pairwise } \\
\text { simirarity score }\end{array}$ \\
\hline GO:0009987 cellular process & GO:0043229 intracellular organelle & 0.045 \\
\hline GO:003299I macromolecular complex & GO:0043226 organelle & \\
\hline \multicolumn{3}{|l|}{ GO:0044445 cytosolic part } \\
\hline \multicolumn{3}{|l|}{ GO:0043229 intracellular organelle } \\
\hline \multicolumn{3}{|l|}{ GO:0043226 organelle } \\
\hline GO:003299I macromolecular complex & GO:0016043 cellular component organization & 0.089 \\
\hline GO:00064I 2 translation & GO:0065007 biological regulation & \\
\hline GO:0044267 cellular protein metabolic process & GO:0050789 regulation of biological process & \\
\hline GO:0019538 protein metabolic process & GO:0050794 regulation of cellular process & \\
\hline GO:0044238 primary metabolic process & GO:0009059 macromolecule biosynthetic process & \\
\hline GO:003299I macromolecular complex & GO:0022625 cytosolic large ribosomal subunit & 0.099 \\
\hline GO:0030529 ribonucleoprotein complex & GO:0044424 intracellular part & \\
\hline \multicolumn{3}{|l|}{ GO:0044445 cytosolic part } \\
\hline \multicolumn{3}{|l|}{ GO:0009987 cellular process } \\
\hline \multicolumn{3}{|l|}{ GO:0005840 ribosome } \\
\hline GO:003299I macromolecular complex & GO:0043 I 70 macromolecule metabolic process & 0.091 \\
\hline \multicolumn{3}{|l|}{ GO:0043228 nonmembrane-bounded organelle } \\
\hline \multicolumn{3}{|l|}{ GO:0043232 intracellular nonmembrane- } \\
\hline \multicolumn{3}{|l|}{ bounded organelle } \\
\hline \multicolumn{3}{|l|}{ GO:0044238 primary metabolic process } \\
\hline \multicolumn{3}{|l|}{ GO:0009987 cellular process } \\
\hline GO:003299I macromolecular complex & GO:0043234 protein complex & 0.099 \\
\hline \multicolumn{3}{|l|}{ GO:0009987 cellular process } \\
\hline \multicolumn{3}{|l|}{ GO:0019538 protein metabolic process } \\
\hline \multicolumn{3}{|l|}{ GO:00064I 2 translation } \\
\hline \multicolumn{3}{|l|}{ GO:0043228 nonmembrane-bounded organelle } \\
\hline GO:0009987 cellular process & GO:0010608 posttranscriptional regulation of gene expression & 0.106 \\
\hline GO:0044238 primary metabolic process & GO:00I6070 RNA metabolic process & \\
\hline GO:003299I macromolecular complex & GO:005 I 246 regulation of protein metabolic process & \\
\hline GO:0032268 regulation of cellular protein & GO:00064I7 regulation of translation & \\
\hline metabolic process & GO:0044424 intracellular part & \\
\hline \multicolumn{3}{|l|}{ GO:0044445 cytosolic part } \\
\hline GO:003299I macromolecular complex & - & 0.078 \\
\hline \multicolumn{3}{|l|}{ GO:0009987 cellular process } \\
\hline \multicolumn{3}{|l|}{ GO:0044445 cytosolic part } \\
\hline \multicolumn{3}{|l|}{ GO:0044238 primary metabolic process } \\
\hline GO:00064I 2 translation & & \\
\hline GO:0003674 molecular_function & - & 0.050 \\
\hline GO:0009987 cellular process & & \\
\hline GO:003299I macromolecular complex & GO:005 I 246 regulation of protein metabolic process & 0.057 \\
\hline GO:0032268 regulation of cellular protein & GO:0032268 regulation of cellular protein metabolic process & \\
\hline metabolic process & & \\
\hline GO:0044238 primary metabolic process & & \\
\hline GO:005 I 246 regulation of protein metabolic proc & & \\
\hline GO:0009987 cellular process & & \\
\hline GO:0022627 cytosolic small ribosomal subunit & GO:0030686 90S preribosome & 0.083 \\
\hline GO:003299I macromolecular complex & GO:0015935 small ribosomal subunit & \\
\hline GO:00I5935 small ribosomal subunit & GO:0044422 organelle part & \\
\hline GO:0044445 cytosolic part & GO:0044446 intracellular organelle part & \\
\hline GO:003068690S preribosome & GO:0022627 cytosolic small ribosomal subunit & \\
\hline GO:003299I macromolecular complex & GO:0015934 large ribosomal subunit & 0.094 \\
\hline GO:0044445 cytosolic part & GO:0044464 cell part & \\
\hline GO:0044238 primary metabolic process & GO:003496 I cellular biopolymer biosynthetic process & \\
\hline GO:0005840 ribosome & GO:0034645 cellular macromolecule biosynthetic process & \\
\hline GO:0030529 ribonucleoprotein complex & GO:0022625 cytosolic large ribosomal subunit & \\
\hline- & - & 0.045 \\
\hline
\end{tabular}


Table S2 (Continued)

\begin{tabular}{llllll}
\hline Bicluster ID & $\begin{array}{l}\text { Number } \\
\text { of genes }\end{array}$ & $\begin{array}{l}\text { Number of } \\
\text { conditions }\end{array}$ & $\begin{array}{l}\text { Average } \\
\text { PCC }\end{array}$ & $\begin{array}{l}\text { The minimum adjusted } \\
\text { P-value of GO enrichment }\end{array}$ & $\begin{array}{l}\text { Number of enriched } \\
\text { GO terms }\end{array}$ \\
\hline 66 & 100 & 32 & 0.72 & $<0.001$ & 8
\end{tabular}

67

68

69

70

71

72

73

74

75

76

77

78
124

III

51

106

46

126

87

112

116

68

86

104
29

37

21

30

12

36

25

30

31

14

20

39
0.71

0.71

0.71

0.71

0.71

$<0.001$

8

$<0.001$

0

17
15

9

0

$<0.001 \quad 21$
18

13

$\begin{array}{lll}0.71<0.001 & 7\end{array}$

$0.71<0.001<3$

$0.71<0.001$

23 


\begin{tabular}{|c|c|c|}
\hline Five most significant GO terms & Five most specific GO terms & $\begin{array}{l}\text { Highest pairwise } \\
\text { simirarity score }\end{array}$ \\
\hline GO:0005I 98 structural molecule activity & - & 0.080 \\
\hline \multicolumn{3}{|l|}{ GO:003299I macromolecular complex } \\
\hline \multicolumn{3}{|l|}{ GO:0044445 cytosolic part } \\
\hline \multicolumn{3}{|l|}{ GO:00064I 2 translation } \\
\hline \multicolumn{3}{|l|}{ GO:0009987 cellular process } \\
\hline GO:003299I macromolecular complex & GO:0010608 posttranscriptional regulation of gene expression & 0.097 \\
\hline GO:0043234 protein complex & GO:00064I7 regulation of translation & \\
\hline GO:0009058 biosynthetic process & GO:0009059 macromolecule biosynthetic process & \\
\hline GO:0009987 cellular process & GO:0043284 biopolymer biosynthetic process & \\
\hline GO:0043284 biopolymer biosynthetic process & GO:0044424 intracellular part & \\
\hline GO:003299I macromolecular complex & - & 0.099 \\
\hline \multicolumn{3}{|l|}{ GO:0044238 primary metabolic process } \\
\hline \multicolumn{3}{|l|}{ GO:00064I 2 translation } \\
\hline \multicolumn{3}{|l|}{ GO:0009987 cellular process } \\
\hline \multicolumn{3}{|l|}{ GO:0043228 nonmembrane-bounded organelle } \\
\hline- & - & 0.059 \\
\hline GO:003299I macromolecular complex & GO:0034960 cellular biopolymer metabolic process & 0.065 \\
\hline GO:0044445 cytosolic part & GO:0009059 macromolecule biosynthetic process & \\
\hline GO:0044267 cellular protein metabolic process & GO:0043284 biopolymer biosynthetic process & \\
\hline GO:0019538 protein metabolic process & GO:0044260 cellular macromolecule metabolic process & \\
\hline GO:0005I 98 structural molecule activity & GO:0043234 protein complex & \\
\hline- & - & 0.047 \\
\hline GO:0009987 cellular process & GO:00I7076 purine nucleotide binding & 0.101 \\
\hline GO:0044238 primary metabolic process & GO:0032553 ribonucleotide binding & \\
\hline GO:0016462 pyrophosphatase activity & GO:0032555 purine ribonucleotide binding & \\
\hline GO:00168I7 "hydrolase activity, acting & GO:0000I66 nucleotide binding & \\
\hline on acid anhydrides" & GO:0017III nucleoside-triphosphatase activity & \\
\hline \multirow{2}{*}{\multicolumn{3}{|c|}{$\begin{array}{l}\text { GO:0016818 "hydrolase activity, acting on acid } \\
\text { anhydrides, in phosphorus-containing anhydrides" }\end{array}$}} \\
\hline & & \\
\hline GO:003299I macromolecular complex & GO:00I6070 RNA metabolic process & 0.070 \\
\hline GO:0009987 cellular process & GO:0043 I70 macromolecule metabolic process & \\
\hline \multicolumn{3}{|l|}{ GO:0044238 primary metabolic process } \\
\hline \multicolumn{3}{|l|}{ GO:0030529 ribonucleoprotein complex } \\
\hline \multicolumn{3}{|l|}{ GO:00I6070 RNA metabolic process } \\
\hline GO:003299I macromolecular complex & GO:0010468 regulation of gene expression & 0.085 \\
\hline GO:00064I 2 translation & GO:00 I0556 regulation of macromolecule biosynthetic process & \\
\hline GO:0044238 primary metabolic process & GO:0010608 posttranscriptional regulation of gene expression & \\
\hline GO:0044424 intracellular part & GO:00064I7 regulation of translation & \\
\hline GO:0009058 biosynthetic process & GO:0044424 intracellular part & \\
\hline GO:003299I macromolecular complex & GO:0005737 cytoplasm & 0.093 \\
\hline GO:0005I 98 structural molecule activity & GO:0043234 protein complex & \\
\hline \multicolumn{3}{|l|}{ GO:0044445 cytosolic part } \\
\hline \multicolumn{3}{|l|}{ GO:0044238 primary metabolic process } \\
\hline \multicolumn{3}{|l|}{ GO:0009987 cellular process } \\
\hline GO:0022627 cytosolic small ribosomal subunit & GO:00I5935 small ribosomal subunit & 0.074 \\
\hline GO:00I5935 small ribosomal subunit & GO:0022627 cytosolic small ribosomal subunit & \\
\hline \multicolumn{3}{|l|}{ GO:00064I 2 translation } \\
\hline \multicolumn{3}{|l|}{ GO:0044445 cytosolic part } \\
\hline \multicolumn{3}{|l|}{ GO:0003735 structural constituent of ribosome } \\
\hline GO:0003674 molecular_function & GO:0022627 cytosolic small ribosomal subunit & 0.052 \\
\hline \multicolumn{3}{|l|}{ GO:0022627 cytosolic small ribosomal subunit } \\
\hline GO:003299I macromolecular complex & & \\
\hline GO:003299I macromolecular complex & GO:0003743 translation initiation factor activity & 0.108 \\
\hline GO:0030529 ribonucleoprotein complex & GO:0045 I82 translation regulator activity & \\
\hline GO:00064I 2 translation & GO:0008I35 "translation factor activity, nucleic acid binding" & \\
\hline GO:0044238 primary metabolic process & GO:00I6070 RNA metabolic process & \\
\hline GO:0008I 35 "translation factor activity, nucleic acid binding" & GO:0034960 cellular biopolymer metabolic process & \\
\hline
\end{tabular}


Table S2 (Continued)

\begin{tabular}{llllll}
\hline Bicluster ID & $\begin{array}{l}\text { Number } \\
\text { of genes }\end{array}$ & $\begin{array}{l}\text { Number of } \\
\text { conditions }\end{array}$ & $\begin{array}{l}\text { Average } \\
\text { PCC }\end{array}$ & $\begin{array}{l}\text { The minimum adjusted } \\
\text { P-value of GO enrichment }\end{array}$ & $\begin{array}{l}\text { Number of enriched } \\
\text { GO terms }\end{array}$ \\
\hline 79 & 90 & 23 & 0.71 & $<0.001$ & 9
\end{tabular}




\begin{tabular}{|c|c|c|}
\hline Five most significant GO terms & Five most specific GO terms & $\begin{array}{l}\text { Highest pairwise } \\
\text { simirarity score }\end{array}$ \\
\hline GO:00064I 2 translation & - & 0.060 \\
\hline \multicolumn{3}{|l|}{ GO:0044267 cellular protein metabolic process } \\
\hline \multicolumn{3}{|l|}{ GO:00I 9538 protein metabolic process } \\
\hline \multicolumn{3}{|l|}{ GO:003299I macromolecular complex } \\
\hline \multicolumn{3}{|l|}{ GO:0005840 ribosome } \\
\hline GO:003299I macromolecular complex & - & 0.078 \\
\hline \multicolumn{3}{|l|}{ GO:0043228 nonmembrane-bounded organelle } \\
\hline \multicolumn{3}{|l|}{ GO:0043232 intracellular nonmembrane- } \\
\hline \multicolumn{3}{|l|}{ bounded organelle } \\
\hline \multicolumn{3}{|l|}{ GO:0005I 98 structural molecule activity } \\
\hline \multicolumn{3}{|l|}{ GO:0030529 ribonucleoprotein complex } \\
\hline GO:003299I macromolecular complex & GO:0022625 cytosolic large ribosomal subunit & 0.067 \\
\hline GO:0044445 cytosolic part & GO:0043234 protein complex & \\
\hline GO:0022625 cytosolic large ribosomal subunit & GO:0043 I 70 macromolecule metabolic process & \\
\hline \multicolumn{3}{|l|}{ GO:0044238 primary metabolic process } \\
\hline \multicolumn{3}{|l|}{ GO:0043283 biopolymer metabolic process } \\
\hline GO:0044238 primary metabolic process & GO:0006 I39 "nucleobase, nucleoside, nucleotide & 0.084 \\
\hline GO:0034960 cellular biopolymer metabolic process & and nucleic acid metabolic process" & \\
\hline GO:0009987 cellular process & GO:0008I52 metabolic process & \\
\hline GO:0043283 biopolymer metabolic process & GO:0043229 intracellular organelle & \\
\hline \multirow{2}{*}{ GO:0044260 cellular macromolecule metabolic process } & GO:0043226 organelle & \\
\hline & GO:0034960 cellular biopolymer metabolic process & \\
\hline GO:003299I macromolecular complex & GO:0005488 binding & 0.091 \\
\hline GO:00064I2 translation & GO:0005622 intracellular & \\
\hline GO:0005I98 structural molecule activity & GO:0022625 cytosolic large ribosomal subunit & \\
\hline GO:0005840 ribosome & GO:0044422 organelle part & \\
\hline GO:0044445 cytosolic part & GO:0044446 intracellular organelle part & \\
\hline GO:003299I macromolecular complex & GO:0009059 macromolecule biosynthetic process & 0.098 \\
\hline GO:0044445 cytosolic part & GO:0043284 biopolymer biosynthetic process & \\
\hline GO:0009058 biosynthetic process & GO:0044424 intracellular part & \\
\hline GO:0044249 cellular biosynthetic process & GO:0044237 cellular metabolic process & \\
\hline GO:00064I 2 translation & GO:0044249 cellular biosynthetic process & \\
\hline GO:0030529 ribonucleoprotein complex & GO:0005622 intracellular & 0.074 \\
\hline GO:0044445 cytosolic part & GO:0022625 cytosolic large ribosomal subunit & \\
\hline \multicolumn{3}{|l|}{ GO:003299I macromolecular complex } \\
\hline \multicolumn{3}{|l|}{ GO:0033279 ribosomal subunit } \\
\hline \multicolumn{3}{|l|}{ GO:0043228 nonmembrane-bounded organelle } \\
\hline GO:0009987 cellular process & & 0.090 \\
\hline \multicolumn{3}{|l|}{ GO:00064I 2 translation } \\
\hline \multicolumn{3}{|l|}{ GO:0044445 cytosolic part } \\
\hline \multicolumn{3}{|l|}{ GO:0044238 primary metabolic process } \\
\hline GO:0010468 regulation of gene expression & GO:0019222 regulation of metabolic process & 0.055 \\
\hline GO:00I0556 regulation of macromolecule & GO:0060255 regulation of macromolecule metabolic process & \\
\hline biosynthetic process & GO:0009889 regulation of biosynthetic process & \\
\hline GO:0060255 regulation of macromolecule & GO:003। 323 regulation of cellular metabolic process & \\
\hline metabolic process & GO:003। 326 regulation of cellular biosynthetic process & \\
\hline \multicolumn{3}{|l|}{ GO:003I 326 regulation of cellular biosynthetic process } \\
\hline \multicolumn{3}{|l|}{ GO:0009889 regulation of biosynthetic process } \\
\hline GO:003299I macromolecular complex & GO:0044422 organelle part & 0.073 \\
\hline GO:0044238 primary metabolic process & GO:0044446 intracellular organelle part & \\
\hline GO:00064I 2 translation & GO:0044260 cellular macromolecule metabolic process & \\
\hline GO:0005840 ribosome & GO:0044237 cellular metabolic process & \\
\hline GO:0003735 structural constituent of ribosome & & \\
\hline
\end{tabular}


Table S2 (Continued)

\begin{tabular}{llllll}
\hline Bicluster ID & $\begin{array}{l}\text { Number } \\
\text { of genes }\end{array}$ & $\begin{array}{l}\text { Number of } \\
\text { conditions }\end{array}$ & $\begin{array}{l}\text { Average } \\
\text { PCC }\end{array}$ & $\begin{array}{l}\text { The minimum adjusted } \\
\text { P-value of GO enrichment }\end{array}$ & $\begin{array}{l}\text { Number of enriched } \\
\text { GO terms }\end{array}$ \\
\hline 89 & 82 & 24 & 0.70 & $<0.001$ & 13
\end{tabular}




\begin{tabular}{|c|c|c|}
\hline Five most significant GO terms & Five most specific GO terms & $\begin{array}{l}\text { Highest pairwise } \\
\text { simirarity score }\end{array}$ \\
\hline GO:0044445 cytosolic part & GO:0009889 regulation of biosynthetic process & 0.060 \\
\hline GO:00064I7 regulation of translation & GO:003 I 323 regulation of cellular metabolic process & \\
\hline GO:0010608 posttranscriptional regulation & GO:003 I 326 regulation of cellular biosynthetic process & \\
\hline of gene expression & GO:0010468 regulation of gene expression & \\
\hline GO:0032268 regulation of cellular protein & GO:00 I0556 regulation of macromolecule biosynthetic & \\
\hline metabolic process & process & \\
\hline \multicolumn{3}{|c|}{ GO:005 I 246 regulation of protein metabolic process } \\
\hline GO:0003674 molecular_function & - & 0.050 \\
\hline \multicolumn{3}{|l|}{ GO:0005I 98 structural molecule activity } \\
\hline \multicolumn{3}{|l|}{ GO:0009987 cellular process } \\
\hline \multicolumn{3}{|l|}{ GO:0044238 primary metabolic process } \\
\hline \multicolumn{3}{|l|}{ GO:0044445 cytosolic part } \\
\hline GO:0044445 cytosolic part & GO:0009059 macromolecule biosynthetic process & 0.088 \\
\hline GO:00064I 2 translation & GO:0043284 biopolymer biosynthetic process & \\
\hline GO:003299I macromolecular complex & GO:0044249 cellular biosynthetic process & \\
\hline \multicolumn{3}{|l|}{ GO:0009987 cellular process } \\
\hline \multicolumn{3}{|l|}{ GO:0044238 primary metabolic process } \\
\hline GO:0009987 cellular process & - & 0.090 \\
\hline \multicolumn{3}{|l|}{ GO:003299I macromolecular complex } \\
\hline \multicolumn{3}{|l|}{ GO:0043228 nonmembrane-bounded organelle } \\
\hline \multicolumn{3}{|l|}{ GO:0043232 intracellular nonmembrane- } \\
\hline \multicolumn{3}{|l|}{ bounded organelle } \\
\hline GO:003299I macromolecular complex & GO:0005083 small GTPase regulator activity & 0.067 \\
\hline GO:00064I7 regulation of translation & GO:0030695 GTPase regulator activity & \\
\hline GO:00I0608 posttranscriptional regulation & GO:0005737 cytoplasm & \\
\hline of gene expression & GO:0010608 posttranscriptional regulation of gene expression & \\
\hline GO:0032268 regulation of cellular protein & GO:005 I 246 regulation of protein metabolic process & \\
\hline \multicolumn{3}{|l|}{ metabolic process } \\
\hline \multicolumn{3}{|l|}{ GO:005 I 246 regulation of protein metabolic process } \\
\hline GO:0009058 biosynthetic process & GO:0019222 regulation of metabolic process & 0.075 \\
\hline GO:0044249 cellular biosynthetic process & GO:0060255 regulation of macromolecule metabolic process & \\
\hline GO:00064I 2 translation & GO:0009889 regulation of biosynthetic process & \\
\hline GO:0009987 cellular process & GO:003 I 323 regulation of cellular metabolic process & \\
\hline GO:0044238 primary metabolic process & GO:003। 326 regulation of cellular biosynthetic process & \\
\hline GO:00064I 2 translation & - & 0.062 \\
\hline \multicolumn{3}{|l|}{ GO:0043228 nonmembrane-bounded organelle } \\
\hline \multicolumn{3}{|l|}{ GO:0043232 intracellular nonmembrane- } \\
\hline \multicolumn{3}{|l|}{ bounded organelle } \\
\hline GO:00064I 2 translation & GO:0034470 ncRNA processing & 0.107 \\
\hline GO:0009987 cellular process & GO:0034660 ncRNA metabolic process & \\
\hline GO:0044238 primary metabolic process & GO:0016070 RNA metabolic process & \\
\hline \multicolumn{3}{|l|}{ GO:0016070 RNA metabolic process } \\
\hline \multicolumn{3}{|l|}{ GO:0034660 ncRNA metabolic process } \\
\hline GO:0003674 molecular_function & - & 0.043 \\
\hline GO:0043228 nonmembrane-bounded organelle & GO:0005575 cellular_component & 0.115 \\
\hline GO:0043232 intracellular nonmembrane- & GO:0044464 cell part & \\
\hline bounded organelle & GO:0010556 regulation of macromolecule biosynthetic process & \\
\hline GO:0005840 ribosome & GO:0005737 cytoplasm & \\
\hline GO:003299I macromolecular complex & GO:00I0608 posttranscriptional regulation of gene expression & \\
\hline \multicolumn{3}{|l|}{ GO:0030529 ribonucleoprotein complex } \\
\hline GO:0005622 intracellular & GO:0005622 intracellular & 0.100 \\
\hline GO:0009987 cellular process & GO:0022627 cytosolic small ribosomal subunit & \\
\hline GO:0044238 primary metabolic process & GO:0032268 regulation of cellular protein & \\
\hline GO:00064I 2 translation & metabolic process & \\
\hline GO:0019538 protein metabolic process & & \\
\hline
\end{tabular}


Table S2 (Continued)

\begin{tabular}{llllll}
\hline Bicluster ID & $\begin{array}{l}\text { Number } \\
\text { of genes }\end{array}$ & $\begin{array}{l}\text { Number of } \\
\text { conditions }\end{array}$ & $\begin{array}{l}\text { Average } \\
\text { PCC }\end{array}$ & $\begin{array}{l}\text { The minimum adjusted } \\
\text { P-value of GO enrichment }\end{array}$ & $\begin{array}{l}\text { Number of enriched } \\
\text { GO terms }\end{array}$ \\
\hline 100 & 110 & 28 & 0.70 & $<0.001$ & 8
\end{tabular}

101

102

98

71

105

140

41

101

99

86

109

110

I I I

98

157
34

28

18

21

32

12

25

21

12

30

15

43
0.69

0.69

0.69

$<0.00$ I

$<0.00$ l

7

0.69

0.69

$<0.00$ I

17

$0.69<0.001$

0.69

$<0.00$ I

38
16

48

I

5

0

24

9 


\begin{tabular}{|c|c|c|}
\hline Five most significant GO terms & Five most specific GO terms & $\begin{array}{l}\text { Highest pairwise } \\
\text { simirarity score }\end{array}$ \\
\hline GO:0009987 cellular process & GO:0044249 cellular biosynthetic process & 0.092 \\
\hline \multicolumn{3}{|l|}{ GO:003299I macromolecular complex } \\
\hline \multicolumn{3}{|l|}{ GO:0044238 primary metabolic process } \\
\hline \multicolumn{3}{|l|}{ GO:0044445 cytosolic part } \\
\hline \multicolumn{3}{|l|}{ GO:0009058 biosynthetic process } \\
\hline GO:0005I 98 structural molecule activity & GO:0044422 organelle part & 0.100 \\
\hline GO:003299I macromolecular complex & GO:0044446 intracellular organelle part & \\
\hline GO:0044445 cytosolic part & GO:005 I 246 regulation of protein metabolic process & \\
\hline GO:00064I 2 translation & GO:00064I7 regulation of translation & \\
\hline GO:0009987 cellular process & GO:0032268 regulation of cellular protein metabolic process & \\
\hline GO:003299I macromolecular complex & GO:0006333 chromatin assembly or disassembly & 0.085 \\
\hline GO:0044238 primary metabolic process & GO:0006446 regulation of translational initiation & \\
\hline GO:00064I 2 translation & GO:0003743 translation initiation factor activity & \\
\hline GO:0043284 biopolymer biosynthetic process & GO:0019222 regulation of metabolic process & \\
\hline GO:0005840 ribosome & GO:0045 I82 translation regulator activity & \\
\hline GO:0003674 molecular_function & - & 0.053 \\
\hline GO:0008I50 biological_process & GO:0043234 protein complex & 0.058 \\
\hline \multicolumn{3}{|l|}{ GO:0009987 cellular process } \\
\hline \multicolumn{3}{|l|}{ GO:0003674 molecular_function } \\
\hline \multicolumn{3}{|l|}{ GO:003299I macromolecular complex } \\
\hline \multicolumn{3}{|l|}{ GO:0043234 protein complex } \\
\hline GO:003299I macromolecular complex & GO:0005575 cellular_component & 0.098 \\
\hline GO:0043234 protein complex & GO:0044464 cell part & \\
\hline GO:0044238 primary metabolic process & GO:00I0608 posttranscriptional regulation of gene expression & \\
\hline GO:0009987 cellular process & GO:0043226 organelle & \\
\hline GO:0044445 cytosolic part & GO:005 I 246 regulation of protein metabolic process & \\
\hline- & - & 0.035 \\
\hline GO:0044238 primary metabolic process & GO:0034645 cellular macromolecule biosynthetic process & 0.080 \\
\hline GO:0005I 98 structural molecule activity & GO:0022625 cytosolic large ribosomal subunit & \\
\hline GO:003299I macromolecular complex & GO:0034960 cellular biopolymer metabolic process & \\
\hline GO:0005840 ribosome & GO:0044260 cellular macromolecule metabolic process & \\
\hline GO:0044445 cytosolic part & GO:0009059 macromolecule biosynthetic process & \\
\hline GO:003299I macromolecular complex & GO:0044424 intracellular part & 0.080 \\
\hline \multicolumn{3}{|l|}{ GO:0019538 protein metabolic process } \\
\hline \multicolumn{3}{|l|}{ GO:0044267 cellular protein metabolic process } \\
\hline \multicolumn{3}{|l|}{ GO:0044238 primary metabolic process } \\
\hline \multicolumn{3}{|l|}{ GO:00064I 2 translation } \\
\hline GO:0044267 cellular protein metabolic process & GO:0043229 intracellular organelle & 0.039 \\
\hline GO:0009987 cellular process & GO:0043226 organelle & \\
\hline \multicolumn{3}{|l|}{ GO:0019538 protein metabolic process } \\
\hline \multicolumn{3}{|l|}{ GO:003299I macromolecular complex } \\
\hline \multicolumn{3}{|l|}{ GO:0043229 intracellular organelle } \\
\hline GO:0043228 nonmembrane-bounded organelle & GO:0044422 organelle part & 0.093 \\
\hline GO:0043232 intracellular nonmembrane-bounded organelle & GO:0044446 intracellular organelle part & \\
\hline GO:0044445 cytosolic part & GO:0044424 intracellular part & \\
\hline \multicolumn{3}{|l|}{ GO:003299I macromolecular complex } \\
\hline \multicolumn{3}{|l|}{ GO:0009987 cellular process } \\
\hline GO:0016043 cellular component organization & GO:0006996 organelle organization & 0.041 \\
\hline GO:0009987 cellular process & GO:00I6043 cellular component organization & \\
\hline \multicolumn{3}{|l|}{ GO:0006996 organelle organization } \\
\hline \multicolumn{3}{|l|}{ GO:003299I macromolecular complex } \\
\hline GO:0008I50 biological_process & & \\
\hline GO:0044238 primary metabolic process & GO:0015934 large ribosomal subunit & 0.108 \\
\hline GO:0030529 ribonucleoprotein complex & GO:0030686 90S preribosome & \\
\hline GO:0009987 cellular process & GO:0044464 cell part & \\
\hline GO:003299I macromolecular complex & GO:003496I cellular biopolymer biosynthetic process & \\
\hline GO:00064I 2 translation & GO:00I5935 small ribosomal subunit & \\
\hline
\end{tabular}


Table S2 (Continued)

\begin{tabular}{llllll}
\hline Bicluster ID & $\begin{array}{l}\text { Number } \\
\text { of genes }\end{array}$ & $\begin{array}{l}\text { Number of } \\
\text { conditions }\end{array}$ & $\begin{array}{l}\text { Average } \\
\text { PCC }\end{array}$ & $\begin{array}{l}\text { The minimum adjusted } \\
\text { P-value of GO enrichment }\end{array}$ & $\begin{array}{l}\text { Number of enriched } \\
\text { GO terms }\end{array}$ \\
\hline 113 & 116 & 34 & 0.68 & $<0.001$ & 21
\end{tabular}

\begin{tabular}{|c|c|c|c|c|}
\hline 114 & 69 & 13 & 0.68 & 0.001 \\
\hline 115 & 96 & 21 & 0.68 & $<0.001$ \\
\hline
\end{tabular}

$\begin{array}{llllll}116 & 38 & 9 & 0.68 & - & 0 \\ 117 & 109 & 30 & 0.68 & <0.001 & 9\end{array}$

118

119

120

$|2|$

122

123

124

125

126

132
17

27

36

16

38

18

31

4 I

25
0.68

0.68

0.68

$<0.00$ I

0.001

0.68

0.68

$<0.001$

0.68

0.67

$0.67<0.001$

$0.67<0.00$ I
8

35

8

27

5

0

9

I

5

38

3

18 


\begin{tabular}{|c|c|c|}
\hline Five most significant GO terms & Five most specific GO terms & $\begin{array}{l}\text { Highest pairwise } \\
\text { simirarity score }\end{array}$ \\
\hline GO:0009058 biosynthetic process & GO:0000 105 histidine biosynthetic process & 0.084 \\
\hline GO:003299I macromolecular complex & GO:0006547 histidine metabolic process & \\
\hline GO:0044249 cellular biosynthetic process & GO:0009075 histidine family amino acid metabolic process & \\
\hline GO:00064I 2 translation & GO:0009076 histidine family amino acid biosynthetic process & \\
\hline GO:0009987 cellular process & GO:0009059 macromolecule biosynthetic process & \\
\hline GO:0009987 cellular process & - & 0.053 \\
\hline GO:0003674 molecular_function & GO:0022627 cytosolic small ribosomal subunit & 0.050 \\
\hline \multicolumn{3}{|l|}{ GO:0009987 cellular process } \\
\hline \multicolumn{3}{|l|}{ GO:0022627 cytosolic small ribosomal subunit } \\
\hline \multicolumn{3}{|l|}{ GO:0044267 cellular protein metabolic process } \\
\hline \multicolumn{3}{|l|}{ GO:0019538 protein metabolic process } \\
\hline- & - & $0.04 I$ \\
\hline GO:003299I macromolecular complex & GO:0043234 protein complex & 0.076 \\
\hline \multicolumn{3}{|l|}{ GO:0044445 cytosolic part } \\
\hline \multicolumn{3}{|l|}{ GO:0009987 cellular process } \\
\hline \multicolumn{3}{|l|}{ GO:00064I 2 translation } \\
\hline \multicolumn{3}{|l|}{ GO:0005I 98 structural molecule activity } \\
\hline GO:0009987 cellular process & - & 0.037 \\
\hline GO:0003674 molecular_function & - & 0.072 \\
\hline \multicolumn{3}{|l|}{ GO:0009987 cellular process } \\
\hline \multicolumn{3}{|l|}{ GO:0044445 cytosolic part } \\
\hline \multicolumn{3}{|l|}{ GO:003299I macromolecular complex } \\
\hline \multicolumn{3}{|l|}{ GO:0008I50 biological_process } \\
\hline GO:0044238 primary metabolic process & GO:0022613 ribonucleoprotein complex biogenesis & 0.097 \\
\hline GO:003299I macromolecular complex & GO:0042254 ribosome biogenesis & \\
\hline GO:0033279 ribosomal subunit & GO:0044085 cellular component biogenesis & \\
\hline GO:0008I52 metabolic process & GO:003496 I cellular biopolymer biosynthetic process & \\
\hline GO:0043283 biopolymer metabolic process & GO:00I5935 small ribosomal subunit & \\
\hline GO:0022627 cytosolic small ribosomal subunit & GO:0043332 mating projection tip & 0.089 \\
\hline GO:0044445 cytosolic part & GO:0044463 cell projection part & \\
\hline GO:003299I macromolecular complex & GO:0022627 cytosolic small ribosomal subunit & \\
\hline \multicolumn{3}{|l|}{ GO:0043332 mating projection tip } \\
\hline \multicolumn{3}{|l|}{ GO:0044463 cell projection part } \\
\hline GO:003299I macromolecular complex & GO:0008I35 "translation factor activity, nucleic acid binding" & 0.106 \\
\hline GO:0044445 cytosolic part & GO:003496 I cellular biopolymer biosynthetic process & \\
\hline GO:00064I 2 translation & GO:0034645 cellular macromolecule biosynthetic process & \\
\hline GO:0009987 cellular process & GO:0043229 intracellular organelle & \\
\hline GO:0043234 protein complex & GO:0044422 organelle part & \\
\hline GO:0003674 molecular_function & GO:0043234 protein complex & 0.053 \\
\hline \multicolumn{3}{|l|}{ GO:0043234 protein complex } \\
\hline \multicolumn{3}{|l|}{ GO:003299I macromolecular complex } \\
\hline GO:003299I macromolecular complex & GO:0005488 binding & 0.093 \\
\hline GO:00064I 2 translation & GO:0044422 organelle part & \\
\hline GO:0009987 cellular process & GO:0044446 intracellular organelle part & \\
\hline \multicolumn{3}{|l|}{ GO:0005488 binding } \\
\hline \multicolumn{3}{|l|}{ GO:0044422 organelle part } \\
\hline GO:0009987 cellular process & GO:00I5935 small ribosomal subunit & 0.092 \\
\hline GO:003299I macromolecular complex & GO:0043229 intracellular organelle & \\
\hline GO:0033279 ribosomal subunit & GO:0044422 organelle part & \\
\hline GO:0044238 primary metabolic process & GO:0044446 intracellular organelle part & \\
\hline GO:00064I 2 translation & GO:0043226 organelle & \\
\hline GO:0044238 primary metabolic process & GO:003 I I 25 rRNA $3^{\prime}$-end processing & 0.080 \\
\hline GO:00I6070 RNA metabolic process & GO:0043628 ncRNA $3^{\prime}$-end processing & \\
\hline GO:0044237 cellular metabolic process & GO:0034660 ncRNA metabolic process & \\
\hline GO:0009987 cellular process & GO:0006I39 "nucleobase, nucleoside, nucleotide & \\
\hline \multirow[t]{2}{*}{ GO:0008I 52 metabolic process } & and nucleic acid metabolic process" & \\
\hline & GO:0008I52 metabolic process & \\
\hline
\end{tabular}


Table S2 (Continued)

\begin{tabular}{llllll}
\hline Bicluster ID & $\begin{array}{l}\text { Number } \\
\text { of genes }\end{array}$ & $\begin{array}{l}\text { Number of } \\
\text { conditions }\end{array}$ & $\begin{array}{l}\text { Average } \\
\text { PCC }\end{array}$ & $\begin{array}{l}\text { The minimum adjusted } \\
\text { P-value of GO enrichment }\end{array}$ & $\begin{array}{l}\text { Number of enriched } \\
\text { GO terms }\end{array}$ \\
\hline 127 & 57 & 14 & 0.67 & - & 0 \\
128 & 51 & 18 & 0.67 & $<0.001$ & 1 \\
129 & 77 & 25 & 0.67 & $<0.001$ & 5
\end{tabular}

130

|3|

132

133

134

135

109

107

28

26

25

35

0.67

0.67

$<0.00$ I

19

24

0.66

0.66

$<0.00$ I

9

16

24

113

0.66

$<0.00$ I

I I

$\begin{array}{ll}- & 0 \\ - & 0\end{array}$ 


\begin{tabular}{|c|c|c|}
\hline Five most significant GO terms & Five most specific GO terms & $\begin{array}{l}\text { Highest pairwise } \\
\text { simirarity score }\end{array}$ \\
\hline- & - & 0.042 \\
\hline GO:0003674 molecular_function & - & 0.044 \\
\hline GO:0009987 cellular process & GO:0034622 cellular macromolecular complex assembly & 0.048 \\
\hline GO:0043933 macromolecular complex & GO:0043933 macromolecular complex subunit organization & \\
\hline subunit organization & GO:003462I cellular macromolecular complex subunit & \\
\hline GO:003462I cellular macromolecular complex & organization & \\
\hline \multicolumn{3}{|l|}{ subunit organization } \\
\hline \multicolumn{3}{|l|}{ GO:0003674 molecular_function } \\
\hline \multicolumn{3}{|l|}{ GO:0034622 cellular macromolecular } \\
\hline \multicolumn{3}{|l|}{ complex assembly } \\
\hline GO:0044238 primary metabolic process & GO:0016070 RNA metabolic process & 0.067 \\
\hline \multicolumn{3}{|l|}{ GO:0016070 RNA metabolic process } \\
\hline \multicolumn{3}{|l|}{ GO:0003674 molecular_function } \\
\hline \multicolumn{3}{|l|}{ GO:0043283 biopolymer metabolic process } \\
\hline GO:0003674 molecular_function & GO:0043229 intracellular organelle & 0.076 \\
\hline GO:0043229 intracellular organelle & GO:0043226 organelle & \\
\hline \multicolumn{3}{|l|}{ GO:003299I macromolecular complex } \\
\hline \multicolumn{3}{|l|}{ GO:0043226 organelle } \\
\hline \multicolumn{3}{|l|}{ GO:0044238 primary metabolic process } \\
\hline GO:003299I macromolecular complex & GO:0005488 binding & 0.097 \\
\hline GO:0044238 primary metabolic process & GO:0005622 intracellular & \\
\hline GO:0009987 cellular process & GO:0044424 intracellular part & \\
\hline GO:0044445 cytosolic part & GO:0044249 cellular biosynthetic process & \\
\hline \multicolumn{3}{|l|}{ GO:0005I98 structural molecule activity } \\
\hline GO:003299I macromolecular complex & GO:003496I cellular biopolymer biosynthetic process & 0.096 \\
\hline GO:00064I2 translation & GO:0034645 cellular macromolecule biosynthetic process & \\
\hline GO:0005I 98 structural molecule activity & GO:0009059 macromolecule biosynthetic process & \\
\hline GO:0005840 ribosome & GO:0043284 biopolymer biosynthetic process & \\
\hline GO:0043284 biopolymer biosynthetic process & GO:0043234 protein complex & \\
\hline GO:0005I 98 structural molecule activity & GO:0005737 cytoplasm & 0.074 \\
\hline GO:0043228 nonmembrane-bounded organelle & GO:00I5935 small ribosomal subunit & \\
\hline GO:0043232 intracellular nonmembrane- & GO:0022627 cytosolic small ribosomal subunit & \\
\hline bounded organelle & GO:0032268 regulation of cellular protein metabolic process & \\
\hline \multicolumn{3}{|l|}{ GO:0044445 cytosolic part } \\
\hline \multicolumn{3}{|l|}{ GO:0005840 ribosome } \\
\hline GO:0009058 biosynthetic process & GO:0003676 nucleic acid binding & 0.078 \\
\hline GO:0044238 primary metabolic process & GO:0006I39 "nucleobase, nucleoside, nucleotide and nucleic & \\
\hline GO:0044249 cellular biosynthetic process & acid metabolic process" & \\
\hline GO:003299I macromolecular complex & GO:0008I52 metabolic process & \\
\hline \multirow[t]{2}{*}{ GO:0043284 biopolymer biosynthetic process } & GO:00064I7 regulation of translation & \\
\hline & GO:0009059 macromolecule biosynthetic process & \\
\hline GO:0000462 "maturation of SSU-rRNA from & GO:0000462 "maturation of SSU-rRNA from tricistronic & 0.050 \\
\hline tricistronic rRNA transcript (SSU-rRNA, & rRNA transcript (SSU-rRNA, 5.8S rRNA, LSU-rRNA)" & \\
\hline 5.8S rRNA, LSU-rRNA)" & GO:0030490 maturation of SSU-rRNA & \\
\hline GO:0030490 maturation of SSU-rRNA & GO:0022627 cytosolic small ribosomal subunit & \\
\hline \multicolumn{3}{|l|}{ GO:0022627 cytosolic small ribosomal subunit } \\
\hline \multicolumn{3}{|l|}{ GO:00064I 2 translation } \\
\hline \multicolumn{3}{|l|}{ GO:0043228 nonmembrane-bounded organelle } \\
\hline GO:0044238 primary metabolic process & GO:0008I52 metabolic process & 0.080 \\
\hline GO:0030529 ribonucleoprotein complex & GO:0044237 cellular metabolic process & \\
\hline \multicolumn{3}{|l|}{ GO:0005840 ribosome } \\
\hline \multicolumn{3}{|l|}{ GO:0043228 nonmembrane-bounded organelle } \\
\hline GO:0043232 intracellular nonmembrane- & & \\
\hline bounded organelle & & \\
\hline- & - & 0.033 \\
\hline- & - & 0.041 \\
\hline
\end{tabular}


Table S2 (Continued)

\begin{tabular}{llllll}
\hline Bicluster ID & $\begin{array}{l}\text { Number } \\
\text { of genes }\end{array}$ & $\begin{array}{l}\text { Number of } \\
\text { conditions }\end{array}$ & $\begin{array}{l}\text { Average } \\
\text { PCC }\end{array}$ & $\begin{array}{l}\text { The minimum adjusted } \\
\text { P-value of GO enrichment }\end{array}$ & $\begin{array}{l}\text { Number of enriched } \\
\text { GO terms }\end{array}$ \\
\hline 140 & 135 & 37 & 0.66 & $<0.001$ & 14
\end{tabular}

\begin{tabular}{lllll}
151 & 61 & 21 & 0.65 & $<0.001$ \\
152 & 62 & 15 & 0.65 & $<0.001$ \\
\hline
\end{tabular}




\begin{tabular}{|c|c|c|}
\hline Five most significant GO terms & Five most specific GO terms & $\begin{array}{l}\text { Highest pairwise } \\
\text { simirarity score }\end{array}$ \\
\hline GO:0044238 primary metabolic process & GO:0005488 binding & 0.101 \\
\hline GO:003299I macromolecular complex & GO:0044424 intracellular part & \\
\hline GO:0009987 cellular process & GO:0044237 cellular metabolic process & \\
\hline GO:0043228 nonmembrane-bounded organelle & GO:0043 I 70 macromolecule metabolic process & \\
\hline \multicolumn{3}{|l|}{ GO:0043232 intracellular nonmembrane- } \\
\hline \multicolumn{3}{|l|}{ bounded organelle } \\
\hline GO:0009987 cellular process & GO:0065007 biological regulation & 0.063 \\
\hline GO:0043228 nonmembrane-bounded organelle & GO:0050789 regulation of biological process & \\
\hline GO:0043232 intracellular nonmembrane-bounded & GO:0050794 regulation of cellular process & \\
\hline organelle & GO:0043229 intracellular organelle & \\
\hline GO:0043229 intracellular organelle & GO:0043226 organelle & \\
\hline \multicolumn{3}{|l|}{ GO:0043226 organelle } \\
\hline GO:0044238 primary metabolic process & GO:0003824 catalytic activity & 0.091 \\
\hline GO:003299I macromolecular complex & GO:0006396 RNA processing & \\
\hline GO:0009987 cellular process & GO:0030684 preribosome & \\
\hline GO:0006396 RNA processing & GO:0030686 preribosome & \\
\hline GO:00I6070 RNA metabolic process & GO:0034470 ncRNA processing & \\
\hline GO:003299I macromolecular complex & & 0.064 \\
\hline GO:0019538 protein metabolic process & GO:0008I52 metabolic process & \\
\hline GO:0044238 primary metabolic process & GO:0034960 cellular biopolymer metabolic process & \\
\hline GO:0043283 biopolymer metabolic process & GO:0044260 cellular macromolecule metabolic process & \\
\hline \multirow[t]{2}{*}{ GO:0044267 cellular protein metabolic process } & GO:0044237 cellular metabolic process & \\
\hline & GO:0043234 protein complex & \\
\hline GO:0009987 cellular process & - & 0.052 \\
\hline \multicolumn{3}{|l|}{ GO:0003674 molecular_function } \\
\hline \multicolumn{3}{|l|}{ GO:0008I50 biological_process } \\
\hline GO:0008I50 biological_process & - & 0.045 \\
\hline \multicolumn{3}{|l|}{ GO:0003674 molecular_function } \\
\hline GO:003299I macromolecular complex & GO:0009059 macromolecule biosynthetic process & 0.061 \\
\hline GO:0044238 primary metabolic process & GO:0043284 biopolymer biosynthetic process & \\
\hline GO:0009058 biosynthetic process & GO:0044249 cellular biosynthetic process & \\
\hline \multicolumn{3}{|l|}{ GO:0044249 cellular biosynthetic process } \\
\hline \multicolumn{3}{|l|}{ GO:0009987 cellular process } \\
\hline GO:0003824 catalytic activity & GO:0003824 catalytic activity & 0.088 \\
\hline GO:003299I macromolecular complex & GO:0030684 preribosome & \\
\hline \multicolumn{3}{|l|}{ GO:0044238 primary metabolic process } \\
\hline \multicolumn{3}{|l|}{ GO:0030684 preribosome } \\
\hline GO:0044238 primary metabolic process & GO:0000459 exonucleolytic trimming during rRNA processing & 0.070 \\
\hline GO:0034660 ncRNA metabolic process & GO:0000467 "exonucleolytic trimming to generate & \\
\hline GO:0034470 ncRNA processing & mature $3^{\prime}$-end of $5.8 \mathrm{~S}$ rRNA from tricistronic rRNA & \\
\hline GO:003 I I 25 rRNA 3'-end processing & transcript (SSU-rRNA, 5.8S rRNA, LSU-rRNA)" & \\
\hline \multirow[t]{3}{*}{ GO:0009987 cellular process } & GO:0000469 cleavages during rRNA processing & \\
\hline & GO:0006364 rRNA processing & \\
\hline & GO:0016072 rRNA metabolic process & \\
\hline GO:0044238 primary metabolic process & GO:0044464 cell part & 0.069 \\
\hline GO:0019538 protein metabolic process & GO:0005737 cytoplasm & \\
\hline GO:0044267 cellular protein metabolic process & GO:00064I7 regulation of translation & \\
\hline GO:003299I macromolecular complex & GO:0032268 regulation of cellular protein metabolic process & \\
\hline GO:0005737 cytoplasm & GO:0043 I 70 macromolecule metabolic process & \\
\hline GO:0019538 protein metabolic process & GO:0022625 cytosolic large ribosomal subunit & 0.079 \\
\hline GO:003299I macromolecular complex & GO:0043234 protein complex & \\
\hline \multicolumn{3}{|l|}{ GO:0044267 cellular protein metabolic process } \\
\hline \multicolumn{3}{|l|}{ GO:0044445 cytosolic part } \\
\hline \multicolumn{3}{|l|}{ GO:0005I 98 structural molecule activity } \\
\hline GO:0003674 molecular_function & - & 0.051 \\
\hline GO:0003674 molecular_function & - & $0.04 \mathrm{I}$ \\
\hline
\end{tabular}


Table S2 (Continued)

\begin{tabular}{llllll}
\hline Bicluster ID & $\begin{array}{l}\text { Number } \\
\text { of genes }\end{array}$ & $\begin{array}{l}\text { Number of } \\
\text { conditions }\end{array}$ & $\begin{array}{l}\text { Average } \\
\text { PCC }\end{array}$ & $\begin{array}{l}\text { The minimum adjusted } \\
\text { P-value of GO enrichment }\end{array}$ & $\begin{array}{l}\text { Number of enriched } \\
\text { GO terms }\end{array}$ \\
\hline 153 & 85 & 27 & 0.65 & $<0.001$ & 5
\end{tabular}

154

142

33

0.65

$<0.00$ I

12

155

156

54

71

12

15

34

103

84

19

20

103

74

7

7

6

5

164
57

87

75

56
10
0.65

0.65

$<0.001$

6

$0.65<0.001$

21

$0.65<0.001$

6

$0.65<0.001$

10

0.65

0.001

3

0.64

0.63

0.61

$<0.001$

$<0.00$ I

$<0.00$ I

2

0.54

$-$

0 


\begin{tabular}{|c|c|c|}
\hline Five most significant GO terms & Five most specific GO terms & $\begin{array}{l}\text { Highest pairwise } \\
\text { simirarity score }\end{array}$ \\
\hline GO:0016070 RNA metabolic process & GO:0034660 ncRNA metabolic process & 0.072 \\
\hline GO:0003674 molecular_function & GO:00I6070 RNA metabolic process & \\
\hline \multicolumn{3}{|l|}{ GO:0044238 primary metabolic process } \\
\hline \multicolumn{3}{|l|}{ GO:0009987 cellular process } \\
\hline \multicolumn{3}{|l|}{ GO:0034660 ncRNA metabolic process } \\
\hline GO:0030529 ribonucleoprotein complex & GO:0005622 intracellular & 0.099 \\
\hline GO:0044445 cytosolic part & GO:0043229 intracellular organelle & \\
\hline GO:003299I macromolecular complex & GO:0044422 organelle part & \\
\hline GO:0033279 ribosomal subunit & GO:0044446 intracellular organelle part & \\
\hline GO:0043228 nonmembrane-bounded organelle & GO:0043226 organelle & \\
\hline- & - & 0.039 \\
\hline GO:0043283 biopolymer metabolic process & GO:0034960 cellular biopolymer metabolic process & 0.052 \\
\hline GO:0044238 primary metabolic process & GO:0044260 cellular macromolecule metabolic process & \\
\hline GO:0034960 cellular biopolymer metabolic process & GO:0043I70 macromolecule metabolic process & \\
\hline \multicolumn{3}{|l|}{ GO:0043I70 macromolecule metabolic process } \\
\hline \multicolumn{3}{|l|}{ GO:0044260 cellular macromolecule metabolic process } \\
\hline GO:003299I macromolecular complex & GO:00I5934 large ribosomal subunit & 0.079 \\
\hline GO:0009987 cellular process & GO:0022625 cytosolic large ribosomal subunit & \\
\hline GO:0044445 cytosolic part & GO:005 I 246 regulation of protein metabolic process & \\
\hline GO:0043228 nonmembrane-bounded organelle & GO:0044424 intracellular part & \\
\hline GO:0043232 intracellular nonmembrane- & GO:0032268 regulation of cellular protein & \\
\hline bounded organelle & metabolic process & \\
\hline GO:0005I 98 structural molecule activity & GO:0005488 binding & 0.074 \\
\hline \multicolumn{3}{|l|}{ GO:0005488 binding } \\
\hline \multicolumn{3}{|l|}{ GO:0044445 cytosolic part } \\
\hline \multicolumn{3}{|l|}{ GO:0009987 cellular process } \\
\hline \multicolumn{3}{|l|}{ GO:003299I macromolecular complex } \\
\hline GO:003299I macromolecular complex & GO:0065003 macromolecular complex assembly & 0.063 \\
\hline GO:003462I cellular macromolecular complex & GO:0034622 cellular macromolecular complex assembly & \\
\hline subunit organization & GO:0043933 macromolecular complex subunit organization & \\
\hline GO:0044238 primary metabolic process & GO:003462I cellular macromolecular complex subunit & \\
\hline GO:0009987 cellular process & organization & \\
\hline \multicolumn{3}{|l|}{ GO:0043933 macromolecular complex subunit } \\
\hline \multicolumn{3}{|l|}{ organization } \\
\hline GO:0044422 organelle part & GO:0044422 organelle part & 0.037 \\
\hline GO:0044446 intracellular organelle part & GO:0044446 intracellular organelle part & \\
\hline \multicolumn{3}{|l|}{ GO:0009987 cellular process } \\
\hline GO:0003674 molecular_function & - & 0.048 \\
\hline GO:0003674 molecular_function & - & 0.048 \\
\hline GO:003299I macromolecular complex & - & 0.045 \\
\hline \multicolumn{3}{|l|}{ GO:0003674 molecular_function } \\
\hline- & - & 0.033 \\
\hline
\end{tabular}

Notes: The steps to select specific GO terms from each cluster. (I) We hypothesise if a GO term appears on only a small number of biclusters (ie, I of 4 biclusters), it is specific for the biclusters. (2) We have 164 biclusters. By the proportion test, I of 4 biclusters corresponds to $3 \mathrm{I}$ of I 64 biclusters at 0.05 significance level. (3) Therefore, GO terms appear less than 32 times are specific terms.

\section{Publish your work in this journal}

Advances and Applications in Bioinformatics and Chemistry is an international, peer-reviewed open-access journal that publishes articles in the following fields: Computational biomodelling; Bioinformatics; Computational genomics; Molecular modelling; Protein structure modelling and structural genomics; Systems Biology; Computational

\section{Dovepress}

Biochemistry; Computational Biophysics; Chemoinformatics and Drug Design; In silico ADME/Tox prediction. The manuscript management system is completely online and includes a very quick and fair peerreview system, which is all easy to use. Visit http://www.dovepress.com/ testimonials.php to read real quotes from published authors.

Submit your manuscript here: http://www.dovepress.com/advances-and-applications-in-bioinformatics-and-chemistry-journal 